\title{
Functional Materials for Waste-to-Energy Processes in Supercritical Water
}

\author{
Florentina Maxim ${ }^{1, * \mathbb{C}}$, Iuliana Poenaru ${ }^{1}$, Elena Ecaterina Toma ${ }^{1}$, Giuseppe Stefan Stoian ${ }^{1}{ }^{1}$, \\ Florina Teodorescu ${ }^{1}$, Cristian Hornoiu ${ }^{2}$ (1) and Speranta Tanasescu ${ }^{1}$ \\ 1 Laboratory of Chemical Thermodynamics, "Ilie Murgulescu" Institute of Physical Chemistry, \\ Splaiul Independentei 202, 060021 Bucharest, Romania; ipoenaru@icf.ro (I.P.); \\ tina_toma99@yahoo.com (E.E.T.); stoian.giuseppe95@gmail.com (G.S.S.); fteodorescu@icf.ro (F.T.); \\ stanasescu@icf.ro (S.T.) \\ 2 Laboratory of Chemical Kinetics, "Ilie Murgulescu" Institute of Physical Chemistry, \\ Splaiul Independentei 202, 060021 Bucharest, Romania; chornoiu@icf.ro \\ * Correspondence: fmaxim@icf.ro
}

check for updates

Citation: Maxim, F.; Poenaru, I.; Toma, E.E.; Stoian, G.S.; Teodorescu,

F.; Hornoiu, C.; Tanasescu, S.

Functional Materials for

Waste-to-Energy Processes in Supercritical Water. Energies 2021, 14, 7399. https://doi.org/10.3390/ en14217399

Academic Editor: Francisco Javier Gutiérrez Ortiz

Received: 5 October 2021

Accepted: 1 November 2021

Published: 5 November 2021

Publisher's Note: MDPI stays neutral with regard to jurisdictional claims in published maps and institutional affiliations.

Copyright: (c) 2021 by the authors. Licensee MDPI, Basel, Switzerland. This article is an open access article distributed under the terms and conditions of the Creative Commons Attribution (CC BY) license (https:// creativecommons.org/licenses/by/ $4.0 /)$.

\begin{abstract}
In response to increasing energy demand, various types of organic wastes, including industrial and municipal wastewaters, or biomass wastes, are considered reliable energy sources. Wastes are now treated in supercritical water (SCW) for non-fossil fuel production and energy recovery. Considering that SCW technologies are green and energetically effective, to implement them on a large scale is a worldwide interest. However, issues related to the stability and functionality of materials used in the harsh conditions of SCW reactors still need to be addressed. Here we present an overview on materials used in the SCW technologies for energy harvesting from wastes. There are catalysts based on metals or metal oxides, and we discuss on these materials' efficiency and selectivity in SCW conditions. We focus on processes relevant to the waste-to-energy field, such as supercritical water gasification (SCWG) and supercritical water oxidation (SCWO). We discuss the results reported, mainly in the last decades in connection to the current concept of supercritical pseudo-boiling ( $\mathrm{PB})$, a phenomenon occurring at the phase change from liquid-like (LL) to gas-like (GL) state of a fluid. This review aims to be a useful database that provides guidelines for the selection of the abovementioned functional materials (catalysts, catalyst supports, and sorbents) for the SCW process, starting from wastes and ending with energy-relevant products.
\end{abstract}

Keywords: supercritical water; waste treatment; supercritical water gasification; supercritical water oxidation; metal catalysts; metal oxides catalysts

\section{Introduction}

Waste is a material or a good that has reached the end of its life cycle, lost its economic value, and the function it had for the owner, and has become a source of pollution. It is obvious that for a sustainable waste management, all three aspects, economic, social, and environmental, should be addressed (see Introduction in [1]). For this, issues related to the generation, separation, collection, treatment, recycling and reuse, and final disposal of various types of wastes must be taken into account by stakeholders and decision makers [2]. In Europe, the waste management aims to be in line with the waste hierarchy, which prioritizes waste prevention, followed by preparing for reuse, recycling, other recovery and finally disposal as the least desirable option. Within the context of a circular economy [3], waste management focuses on promoting waste treatment options able to preserve the value and properties of waste materials by delivering high quality secondary raw materials to the economy [4].

The treatment in supercritical water of wastes is nowadays one of the most efficient options to obtain energy-relevant products and valuable compounds through recycling or recovery. For instance, chemical recycling of plastic wastes, with high conversion of 
polymers into their monomers, is done in supercritical water through a rapid, selective, clean and efficient process, useful for processing technically difficult wastes [5-10]. At the industrial scale, there are already companies exploring the chemical recycling of mixed waste plastic feedstock using supercritical water, as for example ReNewELP [9]. Their motto is: "We achieve in just 20 min what takes nature 200 million years: turning waste plastic into readily usable hydrocarbon feedstock" (https:/ / renewelp.co.uk/technology/ accessed on 22 September 2021). Electronic and electrical wastes (e-waste) can be treated in supercritical fluids for a high recovery rate of metals through environmentally friendly, highly efficient and effective oxidation and extraction processes [11]. For example, $\mathrm{Cu}$ and $\mathrm{Pb}$ were recovered from e-waste with an efficiency of $99.8 \%$ and $80 \%$, respectively, while up to $90 \%$ of $\mathrm{Sn}, \mathrm{Zn}, \mathrm{Cr}, \mathrm{Cd}$, and Mn could be recovered if the e-waste was pre-treated in supercritical water [12].

Proposed initially at the Massachusetts Institute of Technology (MIT) in the 80s, for use on NASA's space facilities, the MODAR process was developed for the treatment of human wastes by oxidation in supercritical water [13], showing the capability of achieving high destruction efficiencies of hazardous organic constituents in a single step [14]. A similar project is supported by the European Space Agency, under the name MELiSSA (MicroEcological Life Support System Alternative). The MELiSSA project aims to fully recycle all the solid organic wastes generated in space, such as human waste, food waste, and non-edible parts of plants, using supercritical water as the reaction medium [15].

In the early 2000s, at the Karlsruhe Institute of Technology, the first dedicated pilot plant for the treatment of agricultural wastes started to demonstrate its capability for gasification of wet biomass under the conditions of supercritical water. Its name is VERENA (acronym for the German expression "experimental facility for the energetic exploitation of agricultural matter"), and it is based on allothermal, continuous flow process using supercritical water as a solvent for the conversion of organic matter into combustible gas [16].

At the Paul Scherrer Institute (PSI), around 2008, the SunCHem process for the production of bio-methane via continuous catalytic SCW gasification of microalgae was proposed $[17,18]$. Microalgae are a promising bio-resource since they do not compete with food crops and show higher photosynthetic efficiency and biomass production rates compared to terrestrial plants. The SunCHem process is a closed-loop system in which all the liquid (rich in nutrients) and gaseous effluents after the SCW gasification are recycled into the photo-bioreactor. Based on this process, the dedicated mobile gasification plant, KONTIC, is now part of the Energy System Integration or ESI Platform at the PSI, a platform that offers suitable infrastructure to test energy production from biomass (biowaste) in all its various configurations (https://www.psi.ch/en/media/esi-platform accessed on 22 September 2021).

The aforementioned processes are possible due to the unique thermodynamic, transport and solvent properties of water above its critical point $\left(T>T_{\mathrm{cr}}=647 \mathrm{~K}, p>p_{\mathrm{cr}}=221 \mathrm{bar}\right)$. With the knowledge delivered through the fundamental research in the field of supercritical fluids, it is now possible to finely tune the properties of water for the optimization of the operating parameters of SCW reactors. As an example, at 230 bar and in a temperature interval of about $6 \mathrm{~K}$, the water density drops by a factor of three, the specific enthalpy increases $\sim 500 \mathrm{~J} \mathrm{~kg}^{-1}$ [19], the self-diffusion coefficient doubles, and the dielectric constant drops to a half [20].

Two processes that occur in SCW are the most relevant for energy harvesting and/or recovery from the waste sources: gasification and oxidation. The main benefit of the gasification in SCW is that the energy-consuming drying pre-treatment of the organic feedstock is omitted when using water as solvent [21]. Oxidation under SCW conditions has the main advantage over classical wet oxidation of decreasing reaction time, as complete destruction of the organic compounds can be achieved in seconds [22]. Although considered a clean and efficient processes, the main obstacle to implementing SCW technologies at large industrial scale is the high operating cost coming from the necessity to work under high 
temperature and high pressure conditions, and from the technical challenges related to corrosion and salt deposition causing the plugging of pipes in SCW plants. Decreasing the temperature of the SCW process, shortening the reaction time to avoid corrosion and developing reliable methods for salt separation and removal from the SCW streams are the solutions to overcome this obstacle.

Materials employed in the SCW processes as catalysts allow cost-efficient operation of SCW reactors at lower temperatures, shorten the reaction time by increasing the process rates, and suppress the formation of by-products while enhancing the selectivity to the specific reactions and products with desirable properties, as for example the elemental composition and the heating value of the combustible gases/fuels. For example, the SCW reforming of glycerol to produce $\mathrm{H}_{2}$-rich combustible gas can be performed at $923 \mathrm{~K}$ when using Ni-based catalyst [23] comparing to a reaction temperature of $1073 \mathrm{~K}$ for the noncatalytic process [24]. Moreover, the $\mathrm{CO}_{2}$ molar yields reached $100 \%$ when the SCWO of phenol, a toxic compound found in the wastewaters, was performed in the presence of a commercial catalyst named CARULITE $\left(\mathrm{MnO}_{2}+\mathrm{CuO}\right.$ on $\left.\mathrm{Al}_{2} \mathrm{O}_{3}\right)$ compared to $\mathrm{CO}_{2}$ yields of no more than $10 \%$ when the reaction is non-catalytic [25].

Heterogeneous as well as homogenous catalysis in SCW are presented as strategies to reduce the high operation cost as main problem in the development of SCW technologies. The main advantage of heterogeneous catalysis in SCW is related to the ability to recover and reuse the catalyst, as the recyclability of homogeneous catalysts is still an issue to be investigated. However, the heterogeneous catalysts' deactivation by sintering and poisoning due to the presence of sulfur, nitrogen, or coke is still a big challenge [26]. For instance, only a few ppm of sulfur could deactivate the Ru-based catalyst used to obtain $\mathrm{CH}_{4}$-rich combustible gas by SCWG of lignin [27] and microalgae [17]. Commercial $\mathrm{ZnO}$ sorbent material has been used for sulfur removal with good performance under SCW conditions [28].

Screening the literature, we found many studies reporting results on catalysis under SCW conditions. Over the years, the major findings of these studies have been summarized and presented as reviews. Catalytic hydrothermal gasification was overviewed in [26,29-33], while the catalytic oxidation in SCW was reviewed in [34,35].

In the present review, we focus on the role of functional materials (catalysts, sorbents, and supports) in the SCW processes starting from wastes and ending with energy-relevant products. First, we start by summarizing the main findings of fundamental research on the structure and thermodynamics of water above the critical point and the water properties' effects on the performance of SCW processes. In the second part, we give a few details about gasification and oxidation in SCW. The final part is dedicated to overview the main studies reporting results on metal and metal oxides used as active materials for catalysis or adsorption in SCWG and SCWO.

\section{Supercritical Water as Reaction Medium}

First, it is important to note that, when issues related to the fundamental research on supercritical water are discussed, the acronym used is $\mathrm{scH}_{2} \mathrm{O}[19,36]$, instead of the abbreviation SCW, which is mostly used when referring to the applications of water in supercritical conditions. For being consistent, the abbreviation SCW is used in this review.

Water above the critical point has been described in textbooks as a single phase of a fluid with properties highly sensitive to pressure. During isobaric heating, while the density of SCW decreases, the features of the solution change from those of an aqueous solution to those of a non-aqueous solution and eventually to a gaseous solution. In consequence, it is expected that the reaction mechanisms change from those involving ions to those involving radicals [36] (p. 120).

The supercritical region in the phase diagram of a fluid is divided into two different regimes, the liquid-like (LL) and the gas-like (GL), by the transitional lines most frequently named Frenkel and Widom [37-40]. The transition at the Frenkel line is dynamic, and across this line the LL and GL phases are different in terms of diffusion mechanisms [38,41]. Across 
the Widom line (or Nishikawa's ridge [42,43]) the transition is purely thermodynamic, and relates to the thermodynamic anomalies in the fluid's critical behavior. For instance, various thermodynamic quantities, such as the heat capacity, compressibility coefficient, thermal expansion coefficient, and the density fluctuations go through maxima upon varying pressure or temperature. The locations of those maxima form a whole set of 'lines of maxima' in the supercritical region and all these lines merge asymptotically into a single line when approaching the critical point [38]. In particular, the Widom line depends on the used quantity and has an upper pressure limit between 3 and 10 times higher than the critical pressure [44].

Across the Widom line at which is located the maxima of isobaric heat capacity, the phenomenon of supercritical pseudo-boiling (PB) occurs when the fluid changes its state from LL to GL phase [45]. Therefore, this transitional line is referred to as PB-Widom line [19]. The PB transition obeys similar laws to sub-critical boiling [46] having associated with it a large heat capacity and a steep decay in fluid's density. However, the PB is a continuous transition, happening at a finite temperature interval in which the energy supplied to the system is used to both increase the temperature of the fluid and change its structure from LL to GL states [45]. It was demonstrated that, during isobaric heating in the supercritical region closed to the critical point, while the water density drops by a factor of three in the PB transitional region, the system needs more than 16 times less energy to increase its temperature by $1 \mathrm{~K}$ than to overcome the molecular attractions at the structural change from LL to GL phase [19].

In Figure 1 we present the water density variation in the supercritical region, along isobars and in the temperature interval from 600 to $800 \mathrm{~K}$ that includes the PB transitional region. We use the density values reported in the NIST database [47]. It is to note in Figure 1, that the density decay within the $\mathrm{PB}$ region is smothering as the pressure increases. Additionally, Figure 1 shows how the water density varies along the PB-Widom line (red line) that is determined based on Banuti's theory of PB [45] and using NIST reference data for water heat capacity [47] (see Experimental section in [19]). It can be seen that the water density value at the PB-Widom line increases as pressure increase, however, it is always close to the critical density of $322 \mathrm{~kg} \mathrm{~m}^{-3}$. It is important to remember that water has LL densities above the PB-Widom line, while below this line, GL water density values are recorded [19].

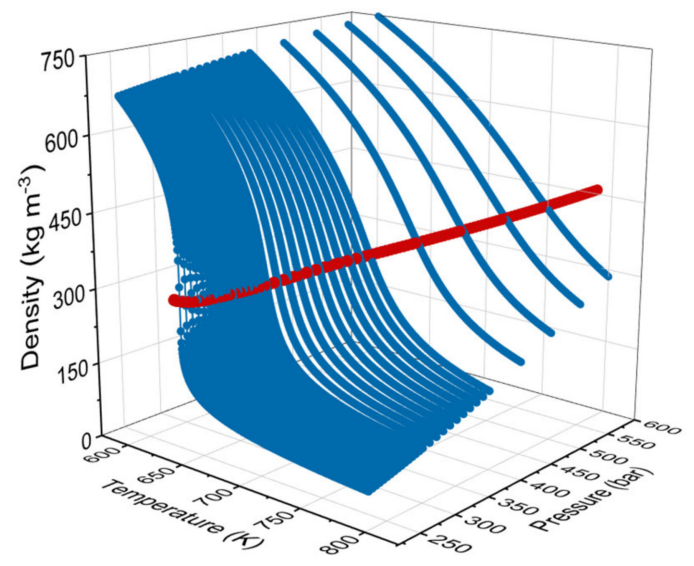

Figure 1. Water density variation with temperature along isobars in the PB region; the red line corresponds to the density values at the PB-Widom line calculated based on Banuti's theory [45]; Water's density values on the blue curves have been obtained from publicly available NIST database at https: / / webbook.nist.gov / chemistry / fluid (last accessed on 28 October 2021).

Maxim et al. studied the PB phenomenon by neutron imaging [19,48], which is a sensitive experimental technique for water density analysis. Results presented in [48] are the first reporting on the experimental visualization of SCW pseudo-boiling, providing 
evidence of how the system evolves rapidly from the LL to the GL phase of water as the PB-Widom line is crossed during isobaric heating. Moreover, it is shown from the reported neutron imaging density maps that the LL and GL states can be identified macroscopically when SCW interacts with microporous carbon with a monolithic structure [48]. The main results of this study are presented as a summary in Figure 2 that shows the water density fluctuations in the presence of carbon porous monolith when the SCW reactor is heated at 225 bar. It is visible that the LL and GL phases can be separated at the time of PB. Moreover, in a temperature range of a few $\mathrm{K}$, which is the PB region, the density of water changes from LL values, of about $650 \mathrm{~kg} \mathrm{~m}^{-3}$, to values for GL close to $150 \mathrm{~kg} \mathrm{~m}^{-3}$ [48].
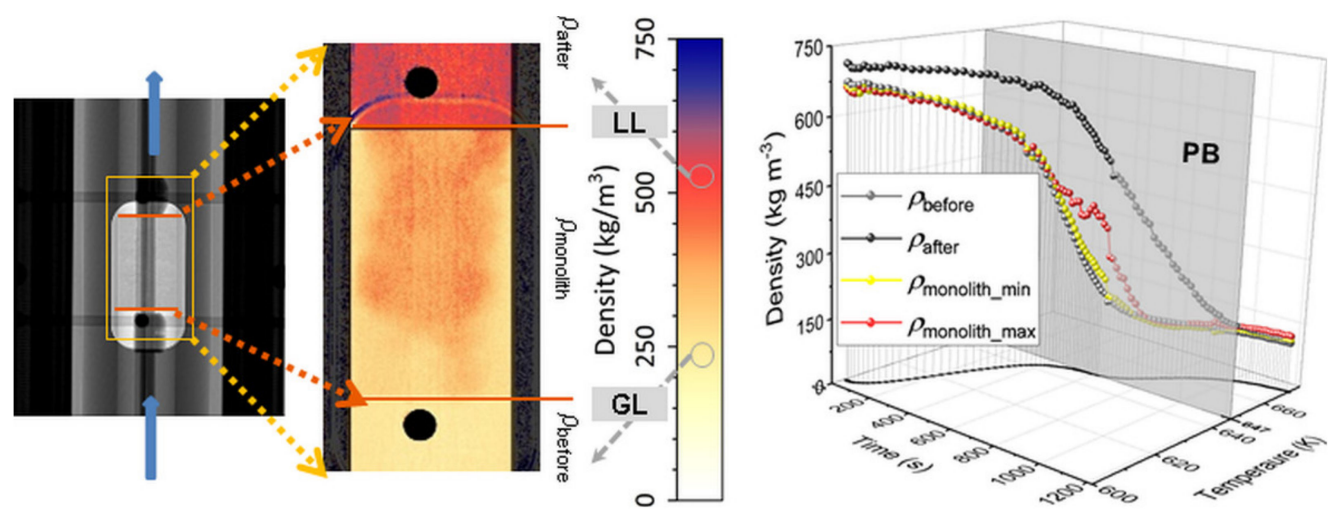

Figure 2. Water density fluctuations in the presence of carbon porous monolith; from left to right: the carbon monolith inside the SCW reactor, the color scaled water density map and the density variation over time; it is visible the LL/GL phase separation at the time of PB (adapted from [48]).

The findings reported in studies by Maxim et al. $[19,48]$ are very important as they managed to finely tune the properties of water, which is of great interest for the optimization of SCW reactors' operating parameters. Water properties strongly affect the reaction rates and selectivity of the organic reactions in SCW. For example, it is reported that the catalytic gasification of lignin is enhanced at $673 \mathrm{~K}$ by the increase in water density. After $180 \mathrm{~min}$, the total gas yield reaches $100 \%$ when the water density is above $330 \mathrm{~kg} \mathrm{~m}^{-3}$ (LL SCW), compared to only $60-70 \%$ when the water density is below $200 \mathrm{~kg} \mathrm{~m}^{-3}$, although the composition of the gas product is independent of the SCW density [49]. At a water density of $330 \mathrm{~kg} \mathrm{~m}^{-3}$ (the value at the PB-Widom line), and at $673 \mathrm{~K}$, after $15 \mathrm{~min}$ of reaction over the catalyst, the carbon yield of the gas products from sugarcane bagasse, cellulose, and lignin was 50.3, 74.4, and $31.1 \mathrm{C} \%$, respectively [50]. The amount of sulfur that remained on the catalyst surface after gasification of lignin at $673 \mathrm{~K}$ for $180 \mathrm{~min}$ decreased with increasing water density from $500 \mathrm{~kg} \mathrm{~m}^{-3}$ to $650 \mathrm{~kg} \mathrm{~m}^{-3}$ [27]. In the case of catalytic gasification of alkylphenols as lignin model compounds at $673 \mathrm{~K}$, for $15 \mathrm{~min}$, the ratio of methane increased with increasing water density in the GL region, from 100 to $300 \mathrm{~kg} \mathrm{~m}^{-3}$ [51]. The water density significantly affects the carbon gasification efficiency of catalytic SCWG at $873 \mathrm{~K}$ with wastewater obtained from the hydrothermal liquefaction of microalgae. It is reported that by increasing the density from $107 \mathrm{~kg} \mathrm{~m}^{-3}$ to $263 \mathrm{~kg} \mathrm{~m}^{-3}$, the carbon gasification efficiency increased from $25.43 \%$ to $97.63 \%$, respectively. The authors claim that the release of more $\mathrm{H} \bullet$ and $\mathrm{OH} \bullet$ radicals over the $\mathrm{Ru} / \mathrm{C}$ catalyst with the water density increase, provides a more effective medium for the decomposition of the organic matter [52]. The acid catalytic properties of metal oxides in SCW depend on the water properties. At $673 \mathrm{~K}$, it was found that the water density has two different effects on model organic reactions rates catalyzed by $\mathrm{TiO}_{2}, \mathrm{Nb}_{2} \mathrm{O}_{5}$, and $\mathrm{NbO}_{X} / \mathrm{TiO}_{2}$. One is a suppression effect with increasing water density from $167 \mathrm{~kg} \mathrm{~m}^{-3}$ to $626 \mathrm{~kg} \mathrm{~m}^{-3}$ caused by competitive adsorption between water and the reactants on acid sites. The other is a promotion effect of Brønsted acidity with increasing water density caused by water dissociation on acid sites due to a large ionic product of water [53]. Screening the literature reporting results on 
SCWO, it is notable that the oxidation in SCW is mainly performed in the GL region, with water densities lower than $200 \mathrm{~kg} \mathrm{~m}^{-3}$ [54].

\section{Supercritical Water Processes Relevant for Waste Treatment}

As stated above, gasification and oxidation performed in water under supercritical conditions are the most applicable processes for waste treatment.

A system working under SCW conditions can be operated only in batches at the lab-scale, or in continuous/plug flow mode, applicable at both lab-scale and at pilot or industrial plants. The advantage of the flow systems over batch processing is the possibility to include engineered mixers for the water and feedstock streams. Comparative studies of waste SCWO experiments have revealed that continuous flow reactors outperform batch reactors in terms of treatment capacity, waste destruction efficiency and reproducibility $[15,55]$.

Schematically, a continuous SCW system can be presented as containing six main sections (Figure 3): (1) feeding, (2) pressurization/depressurization, (3) heating/heat exchange/heat recovery, (4) reactor, (5) phase separation, (6) sampling and characterization.

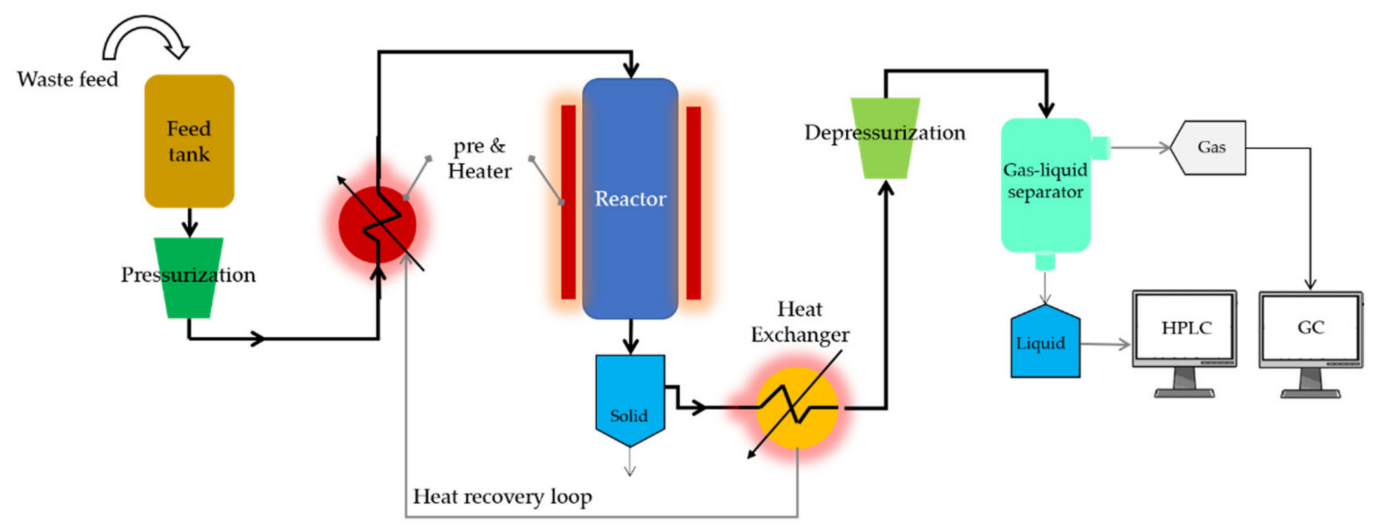

Figure 3. Schematic representation of a continuous flow SCW system.

In the first three sections the operating parameters, such as feed concentration, time, pressure, and temperature are established. The flow rate is an important parameter to be set in the feeding section. Based on the amount of feedstock, water and the flow rate, the time parameter is established and is reported as reaction time, residence time, space time or contact time. The pressure inside the batch reactor is auto generated and can be estimated based on pressure-volume-temperature relations [56] taking into account the operating temperature and the reactor's degree of filling [5]. In continuous flow systems pressure control and depressurization are ensured by a back-pressure regulator valve, as for example in SCWG [57], or in SCWO [58-60]. The fourth part, the reactor, is the main component of the SCW system. Reactors must be made of materials able to withstand the high-temperature and high-pressure conditions and to be corrosive resistant. The materials used for SCW systems' reactors are most commonly stainless steel [61-68], Inconel [69], or Hastelloy [70]. At lab scale, for batch SCW systems, quartz bas also been used as a reactor material [71-74]. A ceramic inlay of aluminum oxide has also been proposed for use in SCWG reactors [75]. For continuous systems, tubular reactors with different orientations have been proposed. In the horizontal position $[24,76,77]$, the reactants mixture is more uniformly exposed to the reaction conditions, while the vertical orientation $[17,28,78-82]$ of the reactor is better for salts removal. For the same issue of salts removal, an inclined plug-flow reactor was recently used for the SCWO process [55]. Microchannel reactors (coiled) are also used for SCWG experiments [83,84] and also for SCWO studies [15,54]. In the catalytic processes the catalyst is loaded in the reactor as fixed/packed [85] or fluidized bed [86]. In the fifth section, the process products in gaseous, liquid and solid phases are separated. The last part of the SCW system helps for process performance evaluation 
which is based on sampling and characterization of gas, liquid, and solid products. Most commonly, the products' characterization is performed by on-line techniques, such as gas chromatography (GC), liquid chromatography (LC), total organic carbon (TOC) analysis, etc. The process performance is evaluated in terms of total gas yields, the selectivity towards desirable gas products and the carbon gasification efficiency in the case of SCWG, while for the SCWO the degree of conversion, the total organic carbon, and chemical oxygen demand removal efficiencies can be reported.

\subsection{Supercritical Water Gasification}

The SCWG is a complex process which includes the structural breakdown of the organic matter into low-molecular weight compounds, and the reactions in SCW with the formation of gaseous fraction containing $\mathrm{H}_{2}, \mathrm{CO}, \mathrm{CO}_{2}, \mathrm{CH}_{4}$ and other light hydrocarbons, of the water-soluble compounds contained in the liquid fraction and of the solid byproducts, such char, tar, and salts. The overall gasification reaction is endothermic and therefore the SCW system should be operated at high temperature for process efficiency. Three main categories of reactions are studied for the optimization of operating parameters in the SCWG systems. These are the reforming and the water-gas shift reactions for an $\mathrm{H}_{2}$-rich gas product, and the methanation reactions for the formation of $\mathrm{CH}_{4}$ [26]. The reforming reactions are endothermic; the water-gas shift reaction is mildly exothermic, and the methanation reactions are exothermic [87], suggesting that they are thermodynamically favored at high, moderate, and low temperatures, respectively. For the energy cycle optimization and heat recovery it is important to know the energy of these reactions. The thermodynamic studies of the SCWG process are well reported in the literature by Fiori \& Castello [88] and Hantoko [87]. Their thermodynamic equilibrium calculations are carried out based on Gibbs free energy minimization by using Aspen Plus software. Rorrer and Goodwin group studied the gasification of glucose in SCW at $1023 \mathrm{~K}$ and estimated the standard enthalpy of reforming reaction by Hess' Law to be equal to $114 \mathrm{~kJ} \mathrm{~mol}^{-1}$ based on gas-phase water or $246 \mathrm{~kJ} \mathrm{~mol}^{-1}$ based on liquid-phase water [83]. The same group, performed thermodynamic calculations using ChemCad 6.1 for the enthalpy of phenol and xylose reforming at the same $1023 \mathrm{~K}$, and report values of $472 \mathrm{~kJ} \mathrm{~mol}^{-1}$ and $335 \mathrm{~kJ} \mathrm{~mol}^{-1}$, respectively [84]. Using Aspen Plus software, Guiterez Ortiz et al., estimated the enthalpy variation for glycerol reforming in SCW at $1073 \mathrm{~K}$ and 243 bar to a value of $192 \mathrm{~kJ} \mathrm{~mol}^{-1}$ [89].

As the overall gasification reaction is endothermic, the energy recovery within the gasification process is a key issue for cost-efficient operation of SCW systems. The energy recovery is possible only for systems operated in continuous/plug flow mode [17,79], for example, the VERENA plant used for SCWG of agricultural matter [16]. It was demonstrated that energy yield can reach $85 \%$ if the energy is recovered from hot water at 300 bar and $873 \mathrm{~K}$, while assuming the ideal scenario of no external heat loss [78]. Energy savings of about $15.58 \%$ were achieved when gasification of food waste was performed by a two-step heating process, initiated by a hydrothermal pretreatment for a short time, followed by the SCWG reaction [70].

\subsection{Supercritical Water Oxidation}

The SCWO process is a single-phase reaction between the organic compounds and the oxidant, with the formation of $\mathrm{CO}_{2}$ and $\mathrm{H}_{2} \mathrm{O}$. The heteroatoms, such as chlorine, sulfur, and phosphorus are transformed in inorganic compounds, typically acids, salts, or oxides, while the organic bounded nitrogen usually converts to $\mathrm{N}_{2}$ and $\mathrm{N}_{2} \mathrm{O}$. Unwanted compounds, like dioxins or $\mathrm{NO}_{\mathrm{x}}$ are not normally formed [14,90,91].

Historically, the SCWO emerged as a further development of the wet-air oxidation technology, and as an alternative to the incineration process of the non-biodegradable organic wastes. The main advantages of SCWO over the two above-mentioned processes are the residence time of seconds compared to hours for wet-air oxidation, and lower operating temperature than a typical incinerator. The development of the SCWO technology 
started practically in the early 80 s with the work of Modell for the treatment of sewage sludge [92], and it is based on the MODAR process proposed initially for the treatment of human wastes on NASA' space facilities [13].

As the corrosion and salts deposition are even more challenging issues in the SCWO process, it is mandatory to have a very short reaction/residence time in the reactor and a salt separator section. For this, the SCWO systems differ from SCWGs in the feeding section, as proper mixing and preheating of the feed, water, and oxidant streams should be achieved before the reaction. In this view, feeding systems based on oxidant multiinjection at different reactor points has been proposed to avoid the corrosion and salt deposition problems [93-95]. It was found that oxidant dosage in a SCWO reactor is also an aspect in energy management [93]. Moreover, the multi-injection oxidant feeding systems showed enhanced total organic removal efficiency [94]. In order to overcome corrosion and salt deposition problems, the multi-feed injection technology was coupled with dynamic gas seal wall reactor [95]. An innovative feed system was proposed by Vadillo et al. in 2012 for the treatment of water-insoluble organics and wastewaters with a wide range of concentrations by SCWO at $823 \mathrm{~K}$ and 250 bar. The feed system of the pilot plant located at the University of Cadiz includes three independent feed streams: water, water-insoluble waste and air. The first two streams were mixed under the SCW conditions that allowed complete dissolution of the organic compounds before entering the reactor. The oxidation reactions took place at a high rate and released a significant amount of heat after the addition to the reactor of the third stream, the oxidant. This feed system allows the SCWO process to remain energy self-sufficient [96].

Oxygen is the most used oxidant agent in SCWO [90,92,97-101], which is supplied to the system at various concentrations. Another oxidant used is $\mathrm{H}_{2} \mathrm{O}_{2}[13,15,34,54,55,59,93$, 94,102-108] which during the preheating decomposes and yield molecular oxygen [98,108].

Comparative studies for wastewater treatment by both SCWO and SCWG revealed that the former can be performed in a shorter reaction time $[109,110]$. For instance, from industrial pharmaceutical wastewater treated by SCWG at $673 \mathrm{~K}$, the maximum $\mathrm{H}_{2}$ yield of $126.5 \mathrm{mmol} / \mathrm{L}$ was obtained after $45 \mathrm{~min}$., while after only $5 \mathrm{~min}$, near-complete conversion of $99.5 \%$ was reached by SCWO using $\mathrm{H}_{2} \mathrm{O}_{2}$ and an oxidation coefficient of 4 , at the same operating temperature [110].

\section{Functional Materials for SCW Processes}

The functional materials employed in the SCW processes for waste treatment are manly with catalytic and adsorption activity. This review focuses on metal and metal oxide functional materials for gasification and oxidation in water under supercritical conditions.

\subsection{Metal-Based Catalysts for SCWG}

Catalytic gasification in SCW of different waste feedstocks using metals as the active material is a common practice nowadays for producing combustible gas. This is mainly due to the ability of metals to selectively catalyze the reactions through which $\mathrm{H}_{2}$ is produced, by reforming and water gas shift reactions [111,112] at relatively high temperatures, or by methanation reactions at low SCW temperatures with $\mathrm{CH}_{4}$ production $[50,113,114]$.

Ni-based materials [66,115,116], noble metals [50,117], and some bimetallic catalysts $[112,118,119]$ have been tested for the gasification of various sources of real waste, such as pinewood $[111,120]$ and straw [68,121] biomass, food waste [122], waste cooking oil [123], waste tires [66], and sugarcane bagasse [50]. Table 1 presents a selection of results obtained from the waste gasification experiments under various SCW conditions reported in studies, mostly in recent years.

It was found that Raney $\mathrm{Ni}$ increased the $\mathrm{H}_{2}$ yield by enhancing the water gas shift reaction during the gasification of lignocellulosic biomass, such as sunflower stalk and corncob. At $773 \mathrm{~K}, 314$ bar and after 60 min reaction time, the fraction of $\mathrm{H}_{2}$ obtained from sunflower stalk is two times higher than that produced when corncob is used as feedstock. The catalyst affected both the deamination and the decarboxylation of the amino acids 
during the biomass structural breakdown, as well as degradation of the organic acids to produce mainly $\mathrm{CO}, \mathrm{CO}_{2}, \mathrm{H}_{2}$, and water [115].

Table 1. Metal-based catalysts used for SCWG of waste; there are three categories of metal-based materials included in this table, namely Ni-based, noble metals and bimetallic catalysts.

\begin{tabular}{|c|c|c|c|c|c|}
\hline $\begin{array}{l}\text { Metal } \\
\text { Catalyst }\end{array}$ & Feed & \multicolumn{2}{|c|}{ Optimal Operation Conditions ${ }^{1}$} & $\begin{array}{l}\text { Efficiency and } \\
\text { Selectivity }\end{array}$ & Reference \\
\hline & \multirow{2}{*}{ Raney Ni } & Sunflower stalk & \multirow{2}{*}{$\begin{array}{c}773 \mathrm{~K}, 314 \mathrm{bar} \\
\rho_{\mathrm{H} 2 \mathrm{O}}=123 \mathrm{~kg} \mathrm{~m}^{-3}, \\
60 \mathrm{~min} \text { residence time } \\
8.3 \mathrm{~g} \text { biomass, } 0.8 \mathrm{~g} \text { catalyst }\end{array}$} & $\begin{array}{l}477.3 \mathrm{~g} \text { gas } / \mathrm{kg} \text { biomass } \\
7.9 \mathrm{~mol} \mathrm{H}_{2} / \mathrm{kg} \text { biomass }\end{array}$ & \multirow{2}{*}{$\begin{array}{l}\text { Yanik } \\
\text { et al. 2008 } \\
\text { [115] }\end{array}$} \\
\hline & & Corncob & & $\begin{array}{l}448.1 \mathrm{~g} \text { gas } / \mathrm{kg} \text { biomass } \\
3.47 \mathrm{~mol} \mathrm{H}_{2} / \mathrm{kg} \text { biomass }\end{array}$ & \\
\hline & \multirow{2}{*}{$\begin{array}{c}\mathrm{Ni} / \mathrm{CeO}_{2} / \mathrm{Al}_{2} \mathrm{O}_{3} \\
7.2 \text { wt. \% Ni loading }\end{array}$} & cellulose & \multirow{2}{*}{$\begin{array}{c}823 \mathrm{~K}, \\
30 \mathrm{~min} \text { residence time } \\
\text { water to biomass } 7: 1, \\
0.65 \mathrm{~g} \text { catalyst }\end{array}$} & $\begin{array}{c}24.5 \% \text { carbon efficiency } \\
1.63 \mathrm{mmol}_{2} / \mathrm{g} \\
\text { biomass }\end{array}$ & \multirow{2}{*}{$\begin{array}{l}\text { Ding et al. } \\
2014 \text { [111] }\end{array}$} \\
\hline & & pinewood & & $\begin{array}{l}25.2 \% \text { carbon efficiency } \\
1.3 \mathrm{mmol} \mathrm{H}_{2} / \mathrm{g} \text { biomass }\end{array}$ & \\
\hline & $\begin{array}{l}\text { Ni nanoparticles } \\
\text { (obtained by Ni } \\
\text { precursor }\end{array}$ & $\begin{array}{l}\text { Pinewood } \\
\text { biomass } \\
8996 \text { ppm Ni }\end{array}$ & $\begin{array}{c}773 \mathrm{~K}, \sim 230 \mathrm{bar}^{\prime} \\
\rho_{\mathrm{H} 2 \mathrm{O}}=81 \mathrm{~kg} \mathrm{~m}^{-3} \\
45 \text { min residence time, }\end{array}$ & $\begin{array}{c}19.6 \% \text { carbon } \\
\text { gasification efficiency } \\
9.5 \mathrm{mmol} / \mathrm{g} \text { total gas } \\
\text { yield } \\
2.8 \mathrm{mmol} \mathrm{H}_{2} / \mathrm{g} \text { biomass } \\
\end{array}$ & $\begin{array}{l}\text { Nanda } \\
\text { et al. } 2016\end{array}$ \\
\hline & feedstock material) & $\begin{array}{l}\text { Wheat straw } \\
\text { biomass } \\
12,358 \text { ppm Ni }\end{array}$ & water-to-biomass ratio 10:1 & $\begin{array}{c}32.6 \% \text { carbon } \\
\text { gasification efficiency } \\
5.8 \mathrm{mmol} \mathrm{H}_{2} / \mathrm{g} \text { biomass }\end{array}$ & \\
\hline & $\mathrm{Ni} / \mathrm{Si}-\mathrm{Al}_{2} \mathrm{O}_{3}$ & $\begin{array}{l}\text { Wheat straw } \\
\text { biomass }\end{array}$ & $\begin{array}{c}823 \mathrm{~K}, 230 \mathrm{bar} \\
\rho_{\mathrm{H} 2 \mathrm{O}}=71 \mathrm{~kg} \mathrm{~m}^{-3}, \\
60 \mathrm{~min} \text { reaction time } \\
20 \mathrm{wt} . \% \text { feed concentration, } \\
5 \mathrm{wt} . \% \text { catalyst loading }\end{array}$ & $\begin{array}{c}18.2 \mathrm{mmol} / \mathrm{g} \text { total gas } \\
\text { yield } \\
5.1 \mathrm{mmol} \mathrm{H}_{2} / \mathrm{g} \text { biomass }\end{array}$ & $\begin{array}{l}\text { Nanda } \\
\text { et al. 2018 } \\
\text { [121] }\end{array}$ \\
\hline \multirow[t]{5}{*}{ Ni-based } & $\begin{array}{l}\mathrm{Ni} / \mathrm{ZrO}_{2} \text { modified } \\
10 \text { wt. } \% \text { Ni loading; } \\
\text { Co promoter }\end{array}$ & $\begin{array}{l}\text { Oil-containing } \\
\text { wastewater }\end{array}$ & $\begin{array}{c}823 \mathrm{~K}, \\
30 \text { min retention time } \\
5 \mathrm{wt} . \% \text { feed concentration; } \\
\mathrm{Ni} \text { addition rate of } 10 \%\end{array}$ & $\begin{array}{l}\quad 98.8 \% \text { carbon } \\
\text { gasification efficiency } \\
63.2 \mathrm{~mol} \mathrm{H}_{2} / \mathrm{kg} \text { feed }\end{array}$ & $\begin{array}{l}\text { Kou et al. } \\
2018[124]\end{array}$ \\
\hline & $\mathrm{Ni} / \mathrm{Si}-\mathrm{Al}_{2} \mathrm{O}_{3}$ & Bitumen & $\begin{array}{c}973 \mathrm{~K}, 230 \mathrm{bar}, \\
\rho_{\mathrm{H} 2 \mathrm{O}}=55 \mathrm{~kg} \mathrm{~m}^{-3}, \\
60 \mathrm{~min} \text { reaction time } \\
20 \text { wt. } \% \text { feed concentration, } \\
5 \mathrm{wt} . \% \text { catalyst loading }\end{array}$ & $\begin{array}{c}7.1 \mathrm{mmol} / \mathrm{g} \text { total gas } \\
\text { yield } \\
2.71 \mathrm{mmol} \mathrm{H}_{2} / \mathrm{g} \\
\text { bitumen }\end{array}$ & $\begin{array}{l}\text { Rana et al. } \\
2018 \text { [125] }\end{array}$ \\
\hline & $\mathrm{Ni} / \mathrm{SiO}_{2}-\mathrm{Al}_{2} \mathrm{O}_{3}$ & Waste tires & $\begin{array}{c}898 \mathrm{~K}, 230 \mathrm{bar}^{\prime} \\
\rho_{\mathrm{H} 2 \mathrm{O}}=62 \mathrm{~kg} \mathrm{~m}^{-3}, \\
60 \mathrm{~min} \text { reaction time } \\
5 \text { wt. \% feed concentration, } \\
2 \text { wt. } \% \text { catalyst loading }\end{array}$ & $\begin{array}{l}43.4 \% \text { carbon } \\
\text { gasification efficiency } \\
83.1 \% \mathrm{H}_{2} \text { selectivity }\end{array}$ & $\begin{array}{c}\text { Nanda, } \\
\text { Reddy, } \\
\text { et al. } 2019 \\
\text { [66] }\end{array}$ \\
\hline & $\mathrm{Ni} / \mathrm{Si}-\mathrm{Al}_{2} \mathrm{O}_{3}$ & $\begin{array}{l}\text { Waste cooking } \\
\text { oil }\end{array}$ & $\begin{array}{c}948 \mathrm{~K}, 230 \mathrm{bar}^{\prime} \\
\rho_{\mathrm{H} 2 \mathrm{O}}=57 \mathrm{~kg} \mathrm{~m}^{-3}, \\
60 \mathrm{~min} \text { reaction time } \\
25 \text { wt. } \% \text { feed concentration, } \\
5 \mathrm{wt} \% \% \text { catalyst loading }\end{array}$ & $\begin{array}{c}21.71 \mathrm{~mol} / \mathrm{kg} \text { total gas } \\
\text { yield } \\
74 \% \mathrm{H}_{2} \text { selectivity }\end{array}$ & $\begin{array}{l}\text { Nanda, } \\
\text { Rana, } \\
\text { et al. 2019 } \\
\text { [123] }\end{array}$ \\
\hline & $\begin{array}{c}\mathrm{Ni} / \mathrm{La}-\mathrm{Al}_{2} \mathrm{O}_{3} \\
15 \text { wt. } \% \mathrm{Ni} \text { on support } \\
\text { with } 9 \text { wt. } \% \text { La- } \mathrm{Al}_{2} \mathrm{O}_{3}\end{array}$ & Food waste & $\begin{array}{c}753 \mathrm{~K}, 280 \mathrm{bar} \\
\rho_{\mathrm{H} 2 \mathrm{O}}=113 \mathrm{~kg} \mathrm{~m}^{-3}, \\
40 \mathrm{~min} \text { residence time, } \\
\text { stirring at } 80 \mathrm{rpm} \\
8 \text { wt. } \% \text { feed concentration }\end{array}$ & $\begin{array}{c}20.7 \% \text { carbon } \\
\text { gasification efficiency } \\
8.03 \mathrm{~mol} \mathrm{H}_{2} / \mathrm{kg} \text { food } \\
\text { waste } \\
42.46 \% \mathrm{H}_{2} \text { mole fraction }\end{array}$ & $\begin{array}{l}\text { Su et al. } \\
2020[122]\end{array}$ \\
\hline
\end{tabular}


Table 1. Cont.

\begin{tabular}{|c|c|c|c|c|c|}
\hline \multirow[t]{4}{*}{$\begin{array}{l}\text { Metal } \\
\text { Catalyst }\end{array}$} & Feed & \multicolumn{2}{|c|}{ Optimal Operation Conditions ${ }^{1}$} & \multirow{3}{*}{$\begin{array}{c}\begin{array}{c}\text { Efficiency and } \\
\text { Selectivity }\end{array} \\
\begin{array}{c}4.46 \mathrm{~mol} \mathrm{H}_{2} / \mathrm{kg} \text { gas oil } \\
94 \% \mathrm{H}_{2} \text { selectivity }\end{array} \\
\begin{array}{c}3.68 \mathrm{~mol} \mathrm{H}_{2} / \mathrm{kg} \text { gas oil } \\
78 \% \mathrm{H}_{2} \text { selectivity }\end{array}\end{array}$} & \multirow{3}{*}{$\begin{array}{l}\text { Reference } \\
\text { Rana et al. } \\
2020 \text { [116] }\end{array}$} \\
\hline & \multirow{2}{*}{$\begin{array}{l}\mathrm{Ni} / \text { functionalized } \\
\text { carbon nanotubes } \\
10 \% \mathrm{Ni} \text { loading }\end{array}$} & $\begin{array}{l}\text { Light gas oil } \\
\text { (LGO) }\end{array}$ & \multirow{2}{*}{$\begin{array}{c}948 \mathrm{~K}, 230 \mathrm{bar}^{\prime} \\
\rho_{\mathrm{H} 2 \mathrm{O}}=57 \mathrm{~kg} \mathrm{~m}^{-3} \\
75 \mathrm{~min} \text { reaction time } \\
20 \text { wt. } \% \text { feed concentration, } \\
5 \text { wt. } \% \text { catalyst dosage }\end{array}$} & & \\
\hline & & $\begin{array}{l}\text { Heavy gas oil } \\
\text { (HGO) }\end{array}$ & & & \\
\hline & $\begin{array}{c}\mathrm{Ni} / \mathrm{Ce}-\mathrm{ZrO}_{2} \\
10 \mathrm{wt} \% \mathrm{Ni} \text { loading }\end{array}$ & Soybean straw & $\begin{array}{c}773 \mathrm{~K}, 230-250 \mathrm{bar}^{\prime} \\
\rho_{\mathrm{H} 2 \mathrm{O}}=81-90 \mathrm{~kg} \mathrm{~m}^{-3}, \\
45 \mathrm{~min} \text { residence time } \\
1.11 \mathrm{~g} \text { biomass, } 1 \text { g catalyst, } \\
\text { water to feedstock } 1: 10\end{array}$ & $\begin{array}{l}31.6 \% \text { gasification } \\
\text { efficiency } \\
10.9 \mathrm{mmol} \mathrm{H}_{2} / \mathrm{g} \\
\text { biomass } \\
81.7 \% \mathrm{H}_{2} \text { selectivity }\end{array}$ & $\begin{array}{c}\text { Okolie } \\
\text { et al. } 2021 \\
{[68]}\end{array}$ \\
\hline & 1 Catalyst & Feed & $\begin{array}{l}\text { Optimal Operation } \\
\text { Conditions }{ }^{1}\end{array}$ & $\begin{array}{l}\text { Efficiency and } \\
\text { Selectivity }\end{array}$ & Reference \\
\hline \multirow{7}{*}{$\begin{array}{l}\text { Noble } \\
\text { metals }\end{array}$} & $\begin{array}{c}\mathrm{Ru} / \mathrm{C} \\
2 \% \mathrm{Ru} \text { on } \mathrm{C}\end{array}$ & $\begin{array}{c}\text { Spirulina } \\
\text { platensis algae }\end{array}$ & $\begin{array}{c}675 \mathrm{~K}, 315 \mathrm{bar}, \\
\rho_{\mathrm{H} 2 \mathrm{O}}=390 \mathrm{~kg} \mathrm{~m}^{-3}, 361 \mathrm{~min} \\
\text { reaction time } \\
2.5 \mathrm{wt.} \% \text { feed concentration, } \\
8.1 \mathrm{~g} \text { catalyst } / \mathrm{g} \text { dry matter }\end{array}$ & $\begin{array}{c}109 \% \text { carbon gasification } \\
41.7 \text { vol. } \% \mathrm{CH}_{4} \\
8 \text { vol. } \% \mathrm{H}_{2}\end{array}$ & $\begin{array}{l}\text { Stucki } \\
\text { et al. } 2009 \\
{[79]}\end{array}$ \\
\hline & $\begin{array}{c}\mathrm{Ru} / \mathrm{TiO}_{2} \\
2 \text { wt.\% Ru on } \mathrm{TiO}_{2}\end{array}$ & $\begin{array}{l}\text { Sugarcane } \\
\text { bagasse }\end{array}$ & $\begin{array}{c}673 \mathrm{~K}, 300 \mathrm{bar} \\
\rho_{\mathrm{H} 2 \mathrm{O}}=359 \mathrm{~kg} \mathrm{~m}^{-3}, \\
15 \mathrm{~min} \text { reaction time } \\
0.1 \mathrm{~g} \text { feed, } 0.38 \text { g catalyst }\end{array}$ & $\begin{array}{c}50.3 \% \text { carbon yield } \\
39.4 \% \mathrm{CH}_{4}, 14.4 \% \mathrm{H}_{2}\end{array}$ & $\begin{array}{c}\text { Osada } \\
\text { et al. } 2012 \\
{[50]}\end{array}$ \\
\hline & $\mathrm{Ru} / \mathrm{Al}_{2} \mathrm{O}_{3}$ & $\begin{array}{l}\text { Wheat straw } \\
\text { biomass }\end{array}$ & $\begin{array}{c}823 \mathrm{~K}, 230 \mathrm{bar}, \\
\rho_{\mathrm{H} 2 \mathrm{O}}=71 \mathrm{~kg} \mathrm{~m}^{-3}, \\
60 \mathrm{~min} \text { reaction time } \\
20 \text { wt. } \% \text { feed concentration, } \\
5 \mathrm{wt} . \% \text { catalyst loading }\end{array}$ & $\begin{array}{c}15 \mathrm{mmol} / \mathrm{g} \text { total gas } \\
\text { yield } \\
4.18 \mathrm{mmol} \mathrm{H}_{2} / \mathrm{g} \\
\text { biomass }\end{array}$ & $\begin{array}{l}\text { Nanda } \\
\text { et al. 2018 } \\
\text { [121] }\end{array}$ \\
\hline & $\mathrm{Ru} / \mathrm{Al}_{2} \mathrm{O}_{3}$ & Bitumen & $\begin{array}{c}973 \mathrm{~K}, 230 \mathrm{bar}^{\prime} \\
\rho_{\mathrm{H} 2 \mathrm{O}}=55 \mathrm{~kg} \mathrm{~m}^{-3} \\
60 \mathrm{~min} \text { reaction time } \\
20 \text { wt. } \% \text { feedstock } \\
\text { concentration, } 5 \text { wt. \% } \\
\text { catalyst }\end{array}$ & $\begin{array}{c}6.8 \mathrm{mmol} / \mathrm{g} \text { total gas } \\
\text { yield } \\
2.94 \mathrm{mmol} \mathrm{H}_{2} / \mathrm{g} \\
\text { bitumen }\end{array}$ & $\begin{array}{l}\text { Rana et al. } \\
2018 \text { [125] }\end{array}$ \\
\hline & $\mathrm{Ru} / \mathrm{Al}_{2} \mathrm{O}_{3}$ & Waste tires & $\begin{array}{c}898 \mathrm{~K}, 230-250 \mathrm{bar} \\
\rho_{\mathrm{H} 2 \mathrm{O}}=61.59-67.60 \mathrm{~kg} \mathrm{~m}^{-3}, \\
60 \mathrm{~min} \text { reaction time } \\
5 \text { wt. \% feed concentration, } \\
2 \text { wt. \% catalyst }\end{array}$ & $\begin{array}{c}40.4 \% \text { carbon } \\
\text { gasification efficiency } \\
79.9 \% \mathrm{H}_{2} \text { selectivity }\end{array}$ & $\begin{array}{c}\text { Nanda, } \\
\text { Reddy, } \\
\text { et al. } 2019 \\
\text { [66] }\end{array}$ \\
\hline & $\mathrm{Ru} / \mathrm{Al}_{2} \mathrm{O}_{3}$ & $\begin{array}{l}\text { Waste cooking } \\
\text { oil }\end{array}$ & $\begin{array}{c}948 \mathrm{~K} \sim 230 \mathrm{bar}^{\prime} \\
\rho_{\mathrm{H} 2 \mathrm{O}}=57 \mathrm{~kg} \mathrm{~m}^{-3}, \\
60 \mathrm{~min} \text { reaction time } \\
25 \text { wt. } \% \text { feed concentration, } \\
5 \text { wt. } \% \text { catalyst }\end{array}$ & $\begin{array}{c}21.4 \mathrm{~mol} / \mathrm{kg} \text { total gas } \\
\text { yield } \\
90 \% \mathrm{H}_{2} \text { selectivity }\end{array}$ & $\begin{array}{l}\text { Nanda, } \\
\text { Rana, } \\
\text { et al. 2019 } \\
\text { [123] }\end{array}$ \\
\hline & $\begin{array}{c}\mathrm{Ru} / \mathrm{C} \\
1 \text { wt. } \% \mathrm{Ru} \text { on } \mathrm{C}\end{array}$ & $\begin{array}{l}\text { Wastewater } \\
\text { from } \\
\text { hydrothermal } \\
\text { liquefaction of } \\
\text { microalgae } \\
\text { biomass }\end{array}$ & $\begin{array}{c}873 \mathrm{~K} \sim 460 \mathrm{bar}, \\
\rho_{\mathrm{H} 2 \mathrm{O}}=146 \mathrm{~kg} \mathrm{~m}^{-3}, \\
60 \mathrm{~min} \text { reaction time } \\
12 \mathrm{mg} \text { catalyst } / \mathrm{mL}_{(\mathrm{aq} \cdot)}\end{array}$ & $\begin{array}{l}97.63 \% \text { carbon } \\
\text { gasification efficiency } \\
2.10 \mathrm{mmol} \mathrm{H}_{2} / \mathrm{g}_{(\mathrm{aq} \cdot)}\end{array}$ & $\begin{array}{l}\text { Shan et al. } \\
2021 \text { [52] }\end{array}$ \\
\hline
\end{tabular}


Table 1. Cont.

\begin{tabular}{|c|c|c|c|c|c|}
\hline \multirow[t]{2}{*}{$\begin{array}{l}\text { Metal } \\
\text { Catalyst }\end{array}$} & Feed & \multicolumn{2}{|c|}{ Optimal Operation Conditions ${ }^{1}$} & \multirow{2}{*}{$\begin{array}{c}\begin{array}{c}\text { Efficiency and } \\
\text { Selectivity }\end{array} \\
\qquad \begin{array}{c}66.46 \% \mathrm{H}_{2} \text { efficiency } \\
13.22 \mathrm{mmol} \mathrm{H}_{2} / \mathrm{g} \\
\text { biomass }\end{array}\end{array}$} & \multirow{2}{*}{$\begin{array}{c}\text { Reference } \\
\\
\text { Wang } \\
\text { et al. 2021 } \\
{[74]}\end{array}$} \\
\hline & $\begin{array}{c}\mathrm{K}_{2} \mathrm{CO}_{3}+\underset{\mathrm{Ru}}{\mathrm{A}} / \mathrm{Al}_{2} \mathrm{O}_{3} \\
\end{array}$ & $\begin{array}{l}\text { Depolymerizing } \\
\text { slag }\end{array}$ & $\begin{array}{c}723 \mathrm{~K}, \\
10 \text { min residence time } \\
5 \% \text { feed concentration, } \\
\text { depolymerizing slag, } \\
\mathrm{K}_{2} \mathrm{CO}_{3} \text { and } \mathrm{Ru} / \mathrm{Al}_{2} \mathrm{O}_{3} 2: 1: 1\end{array}$ & & \\
\hline \multirow{5}{*}{ Bimetallics } & $\begin{array}{c}\mathrm{Ni}-\mathrm{Ru} / \mathrm{CeO}_{2} \\
30 \text { wt. } \% \mathrm{Ni}-\mathrm{Ru} \text { on } \\
\mathrm{CeO}_{2} \\
\text { Ni to } \mathrm{Ru} \mathrm{1:0.1}\end{array}$ & $\begin{array}{l}\text { Indole, } \\
\text { nitrogen- } \\
\text { containing } \\
\text { compound }\end{array}$ & $\begin{array}{c}773 \mathrm{~K}, 300 \mathrm{bar}, \\
\rho_{\mathrm{H} 2 \mathrm{O}}=115 \mathrm{~kg} \mathrm{~m}^{-3}, \\
30 \mathrm{~min} \text { reaction time } \\
0.3 \mathrm{~mol} / \mathrm{L} \text { feed } \\
\text { concentration, } 25 \mathrm{wt} . \% \\
\text { catalyst }\end{array}$ & $\begin{array}{c}13.9 \% \text { carbon } \\
\text { gasification efficiency } \\
75 \% \text { C selectivity, } \\
202 \% \text { H selectivity }\end{array}$ & $\begin{array}{l}\text { Guo et al. } \\
2015[118]\end{array}$ \\
\hline & $\begin{array}{c}\mathrm{Fe}-\mathrm{Ni} / \mathrm{Ru}-\mathrm{Al}_{2} \mathrm{O}_{3}(\gamma) \\
12 \text { wt. \% of } \mathrm{Ni} \text { and } \\
6 \mathrm{wt} \% \text { of } \mathrm{Fe} \text { in the } \\
\text { active phase on } \\
\text { support with } 2 \text { wt. \% } \\
\text { of } \mathrm{Ru}-\mathrm{Al}_{2} \mathrm{O}_{3}(\gamma)\end{array}$ & $\begin{array}{l}\text { Enteromorpha } \\
\text { intestinalis algal } \\
\text { biomass }\end{array}$ & $\begin{array}{c}713 \mathrm{~K}, 250 \text { bars, } \\
\rho_{\mathrm{H} 2 \mathrm{O}}=115 \mathrm{~kg} \mathrm{~m}^{-3} \\
10 \mathrm{~min} \text { reaction time } \\
0.06 \mathrm{~g} \text { feed, } 6 \mathrm{~g} \text { water }\end{array}$ & $\begin{array}{c}39 \% \text { carbon gasification } \\
\text { efficiency } \\
12.288 \mathrm{mmol} \mathrm{H}_{2} / \mathrm{g} \\
\text { biomass } \\
74 \% \mathrm{H}_{2} \text { selectivity }\end{array}$ & $\begin{array}{l}\text { Norouzi } \\
\text { et al. } 2017 \\
{[112]}\end{array}$ \\
\hline & \multirow{2}{*}{$\begin{array}{c}\mathrm{Ni}-\mathrm{Ru} / \mathrm{Al}_{2} \mathrm{O}_{3}-\mathrm{ZrO}_{2} \\
10 \text { wt. \% Ni and } \\
0.08 \text { wt. } \% \mathrm{Ru} \text { on } \\
\mathrm{Al}_{2} \mathrm{O}_{3}-\mathrm{ZrO}_{2}\end{array}$} & $\begin{array}{l}\text { Biocrude from } \\
\text { corn husk }\end{array}$ & \multirow{2}{*}{$\begin{array}{c}973 \mathrm{~K}, 250 \mathrm{bar}^{-} \\
\rho_{\mathrm{H} 2 \mathrm{O}}=60 \mathrm{~kg} \mathrm{~m}^{-3}, \\
300 \text { min run time }\end{array}$} & $\begin{array}{l}\sim 90 \% \text { carbon gasification } \\
\text { efficiency } \\
2 \mathrm{~mol} \mathrm{H}_{2} / \mathrm{mol} \mathrm{C} \text { in feed }\end{array}$ & \multirow{2}{*}{$\begin{array}{c}\text { Tushar } \\
\text { et al. 2020 } \\
\text { [126] }\end{array}$} \\
\hline & & $\begin{array}{l}\text { Biocrude from } \\
\text { cattle manure }\end{array}$ & & $\begin{array}{l}\sim 90 \% \text { carbon gasification } \\
\text { efficiency } \\
1 \mathrm{~mol} \mathrm{H}_{2} / \mathrm{mol} \mathrm{C} \text { in feed }\end{array}$ & \\
\hline & $\begin{array}{l}\text { Raney-Ni-Mo } \\
\text { 93.57 wt.\% Raney-Ni } \\
\text { 0.31 wt. \% Mo }\end{array}$ & Sewage sludge & $\begin{array}{c}723 \mathrm{~K}, 286 \mathrm{bar}^{\prime} \\
\rho_{\mathrm{H} 2 \mathrm{O}}=136 \mathrm{~kg} \mathrm{~m}^{-3}, \\
25 \mathrm{~min} \text { residence time } \\
8.9 \text { wt. \% feed concentration, } \\
1.4 \mathrm{~g} \text { catalyst } / \mathrm{g} \text { dry sludge }\end{array}$ & $\begin{array}{c}92 \% \text { gasification } \\
\text { efficiency } \\
18.13 \mathrm{~mol} \mathrm{H}_{2} / \mathrm{kg} \text { dry } \\
\text { sludge }\end{array}$ & $\begin{array}{l}\text { Chen } \\
\text { et al. 2020 } \\
\text { [119] }\end{array}$ \\
\hline
\end{tabular}

${ }^{1}$ Water density values from NIST database at https:/ / webbook.nist.gov/ chemistry/fluid/ (accessed on 28 October 2021).

Ni nanoparticles impregnated on the feedstock material were used as catalyst in the gasification reaction of pinewood and wheat straw biomass. The $\mathrm{H}_{2}$ yield was doubled when the SCWG reaction at $773 \mathrm{~K}$ used wheat straw as the feedstock compared to pinewood [120].

Catalytic activity of $\mathrm{Ni}$ supported on $\mathrm{Si}-\mathrm{Al}_{2} \mathrm{O}_{3}$, was tested at $\mathrm{SCW}$ conditions in the gasification reaction of wheat straw biomass [121], bitumen [125], and waste cooking oil [123]. After $60 \mathrm{~min}$ of reaction time at $230 \mathrm{bar}$, the highest total gas yield was obtained when waste cooking oil was gasified over $\mathrm{Ni} / \mathrm{Si}-\mathrm{Al}_{2} \mathrm{O}_{3}$ at $948 \mathrm{~K}$ [123]. $\mathrm{Ni}$ on $\mathrm{SiO}_{2}-\mathrm{Al}_{2} \mathrm{O}_{3}$ support also exhibited catalytic activity for the SCWG of waste tires at $898 \mathrm{~K}$, with a carbon gasification efficiency as high as $43.4 \%$ [66]. Nearly complete conversion of glycerol (a waste compound from biofuel production) to an $\mathrm{H}_{2}$-rich gas was achieved when commercial $\mathrm{Ni} / \mathrm{Al}_{2} \mathrm{O}_{3}-\mathrm{SiO}_{2}$, was used in the $\mathrm{SCW}$ reforming process [23].

Alumina supported $\mathrm{Ni}$ catalysts, promoted with rare-earth $\mathrm{Ce} / \mathrm{La}$, were used as catalysts in the gasification reaction of pinewood [111] and food waste [122] at $823 \mathrm{~K}$ and $753 \mathrm{~K}$, respectively. The carbon gasification efficiency was around $20 \%$ in both cases, with higher $\mathrm{H}_{2}$ selectivity when an La-promoted $\mathrm{Ni}$ catalyst was used for the food-waste gasification [122] than in the gasification of pinewood over a Ce-promoted Ni catalyst [111]. In the last case, it was found that the small amount of nitrogen and sulfur in the pinewood, contributed to the deactivation of the catalyst and, therefore, low gas yields were obtained [111].

A Ce-promoted $\mathrm{Ni}$ catalyst on $\mathrm{ZrO}_{2}$ support was used for the SCWG of soybean straw at $773 \mathrm{~K}$, and pressure around $240 \mathrm{bar}$. Compared with the performances of other tested 
catalysts in this study, after 45 min residence time, the highest gasification efficiency was obtained with the Ce-promoted $\mathrm{Ni} / \mathrm{ZrO}_{2}$. Moreover, the results highlighted that, besides surface area, coke formation, active metal dispersion, and metal-support interactions are also determining factors in improving product yield and selectivity [68]. The catalytic activity of $\mathrm{Ni} / \mathrm{ZrO}_{2}$ with $\mathrm{Co}$ promoters was tested for the gasification of oil-containing wastewater at $823 \mathrm{~K}$. Carbon gasification efficiency as high as $98.8 \%$ was obtained after $30 \mathrm{~min}$. Actually, it was found that all tested Ni-based catalysts are highly active for the C-C cleavage and water gas shift reaction [124].

The catalytic activity of Ni supported on functionalized carbon nanotubes was evaluated during the gasification in SCW of light and heavy gas oils at $948 \mathrm{~K}$ and 230 bar. It was found that the catalyst improved the $\mathrm{H}_{2}$ gas yield by promoting the cracking and reforming reactions, particularly in the treatment of light gas oil due to the easy accessibility to the active sites [116].

Various noble metals, such as $\mathrm{Ru}, \mathrm{Rh}, \mathrm{Pt}$, and $\mathrm{Pd}$ were tested in catalytic gasification of different feedstocks $[50,117]$. Among these, the Ru-based catalysts were the most active catalysts in the SCWG processes starting from microalgae sources [71,86], sugarcane bagasse [50], or depolymerizing slag [74].

$\mathrm{Ru}$ on an $\mathrm{Al}_{2} \mathrm{O}_{3}$ support demonstrated catalytic activity in the SCWG of wheat straw biomass [121], Canadian Athabasca bitumen [125], waste tires [66], and waste cooking oil [123] at $823 \mathrm{~K}, 973 \mathrm{~K}, 898 \mathrm{~K}$, and $948 \mathrm{~K}$, respectively. The selectivity towards the reactions with $\mathrm{H}_{2}$ as gas product varied from almost $30 \%$, in the case of straw feed [121], to the $90 \%$ achieved when waste cooking oil was the source [123]. The combined use of $\mathrm{K}_{2} \mathrm{CO}_{3}$ and $\mathrm{Ru} / \mathrm{Al}_{2} \mathrm{O}_{3}$ for the SCWG of depolymerizing slag (produced during the process of converting biomass into aircraft fuel, mainly composed of lignin and bits of cellulose and hemicellulose) increased the hydrogen efficiency compared with the non-catalytic process. This is due to a synergetic effect as the active materials act on different reaction stages, the combination performing better together than any of the single catalysts. The $\mathrm{K}_{2} \mathrm{CO}_{3}$ promoted the swelling and hydrolysis of lignocellulose and increased the amounts of phenolic intermediates while $\mathrm{Ru} / \mathrm{Al}_{2} \mathrm{O}_{3}$ facilitated the hydrogenation reaction of hydrolyzed products, ring-opening reactions, and the cleavage of carbon-carbon bonds [74].

$\mathrm{Ru}$ supported on $\mathrm{C}$-based materials was more active for gasification reactions in SCW with formation of $\mathrm{CH}_{4}$ as the main fraction in the gas product. At relatively low temperatures, such as $673 \mathrm{~K}$ and $683 \mathrm{~K}, \mathrm{Ru} / \mathrm{C}$ catalyzed the methanation reaction during the SCWG of Spirulina platensis algae [79] and Nannochloropsis sp. marine microalgae [113], respectively. Complete conversion of sugarcane bagasse to a $\mathrm{CH}_{4}$-rich gas product was achieved when SCWG was performed at $673 \mathrm{~K}$ and 300 bar [50]. A Ru/C catalyst was used for the SCWG processing of wastewater from hydrothermal liquefaction of microalgae biomass at $873 \mathrm{~K}$. With increasing water density and catalyst loading, the production of $\mathrm{CH}_{4}$ and $\mathrm{H}_{2}$ was promoted, with the $\mathrm{H}_{2}$ yield always exceeding the $\mathrm{CH}_{4}$ fraction [52].

The bimetallic $\mathrm{Ni}-\mathrm{Ru}$ on a $\mathrm{CeO}_{2}$ support showed high efficiency and gas yields in producing $\mathrm{CO}_{2}, \mathrm{CH}_{4}$, and $\mathrm{H}_{2}$ from indole, a recalcitrant nitrogen-containing compound related to algal biomass processing. Operating above the critical pressure of water at $723 \mathrm{~K}$ for $30 \mathrm{~min}$ is a prerequisite for the $\mathrm{Ni}-\mathrm{Ru}$ composite catalyst to reach a high conversion of indole [118]. Ni-Ru/ $\mathrm{Al}_{2} \mathrm{O}_{3}-\mathrm{ZrO}_{2}$ catalyst showed significant increase in $\mathrm{H}_{2}$ yield compared to the non-catalytic process in the SCWG of corn husk biocrude at $973 \mathrm{~K}$ and 250 bar. Steep deactivation of the catalyst was observed after $2 \mathrm{~h}$ of steam time. In the same conditions, when used for cattle manure biocrude gasification, it produced much lower and rather steady $\mathrm{H}_{2}$ yield comparing to cornhusk feed. In the 773-973 $\mathrm{K}$ temperature range at 250 bar, the catalyst achieved a gasification efficiency of $\sim 90 \%$ in corn husk and cattle manure biocrude treatment regardless of temperature, producing $\mathrm{H}_{2}$-rich gas [126].

For the SCWG of Enteromorpha intestinalis algae biomass at $713 \mathrm{~K}$ and 250 bar a Rupromoted $\mathrm{Fe}-\mathrm{Ni} / \gamma-\mathrm{Al}_{2} \mathrm{O}_{3}$ nanocatalyst performed better than the un-promoted reference in increasing $\mathrm{H}_{2}$ selectivity and only slightly reduced the gasification efficiency by decreasing 
the amount of $\mathrm{CH}_{4}, \mathrm{CO}$, and $\mathrm{C}_{2}$ hydrocarbons. The Ru-promoted catalyst showed increased dispersion and smaller size $\mathrm{Ni}$ crystallite size $(\sim 3.2 \mathrm{~nm})$ on the $\gamma-\mathrm{Al}_{2} \mathrm{O}_{3}$ support [112].

High hydrogen yield and gasification efficiency was obtained with the Raney-Ni-Mo as catalyst in the SCWG of sewage sludge at $723 \mathrm{~K}$. Compared to other catalysts tested in similar conditions, this bimetallic catalyst had a higher surface area and favorable pore distribution [119].

\subsection{Metal Oxides for SCW Processes}

Table 2 summarizes the main findings of studies reporting results on catalytic SCW processes using metal oxides as the active material.

Table 2. Metal oxide materials for SCW processes.

\begin{tabular}{|c|c|c|c|c|c|}
\hline $\begin{array}{l}\text { SCW Process } \\
\text { Functionality }\end{array}$ & Metal Oxide & Feed & $\begin{array}{l}\text { Optimal Reaction } \\
\text { Conditions }^{1}\end{array}$ & $\begin{array}{l}\text { Efficiency and } \\
\text { Selectivity }\end{array}$ & Reference \\
\hline \multirow{7}{*}{ SCWG catalyst } & \multirow{2}{*}{$\begin{array}{l}\text { Red mud (Fe-oxide } \\
\text { containing residue } \\
\text { from } \\
\text { Al-production) }\end{array}$} & Sunflower stalk & \multirow{2}{*}{$\begin{array}{c}773 \mathrm{~K}, 314 \mathrm{bar}, \\
\rho_{\mathrm{H} 2 \mathrm{O}}=123 \mathrm{~kg} \mathrm{~m}^{-3}, \\
60 \mathrm{~min} \\
8.3 \text { g feed, } 0.8 \text { g catalyst }\end{array}$} & $\begin{array}{c}446.8 \mathrm{~g} \text { gas } / \mathrm{kg} \\
\text { sunflower stalk } \\
8.09 \mathrm{~mol} \mathrm{H}_{2} / \mathrm{kg} \\
\text { sunflower stalk } \\
2.95 \mathrm{~mol} \mathrm{CH}_{4} / \mathrm{kg} \\
\text { sunflower stalk }\end{array}$ & \multirow[t]{2}{*}{$\begin{array}{l}\text { Yanik et al. } \\
2008[115]\end{array}$} \\
\hline & & Corncob & & $\begin{array}{c}426.0 \mathrm{~g} \text { gas } / \mathrm{kg} \text { corncob } \\
5.65 \mathrm{~mol} \mathrm{H} / 2 / \mathrm{kg} \text { corncob } \\
3.33 \mathrm{~mol} \mathrm{CH}_{4} / \mathrm{kg} \\
\text { corncob }\end{array}$ & \\
\hline & $\begin{array}{l}\text { Cubic } \mathrm{CeO}_{2} \\
\text { nanocatalyst }\end{array}$ & Black liquor & $\begin{array}{c}723 \mathrm{~K}, 250 \mathrm{bar} \\
\rho_{\mathrm{H} 2 \mathrm{O}}=109 \mathrm{~kg} \mathrm{~m}^{-3}, \\
60 \text { min reaction time } \\
10 \text { wt. } \% \text { feed } \\
\text { concentration, } 5 \mathrm{~g} \\
\text { catalyst } / \mathrm{g} \text { feed }\end{array}$ & $\begin{array}{l}\sim 20 \% \text { carbon conversion } \\
360 \mu \mathrm{mol} \mathrm{H}_{2}\end{array}$ & $\begin{array}{l}\text { Boucard } \\
\text { et al. } 2015 \\
\text { [127] }\end{array}$ \\
\hline & $\begin{array}{c}\mathrm{RuO}_{2} / \gamma-\mathrm{Al}_{2} \mathrm{O}_{3} \\
20 \text { wt. } \% \mathrm{RuO}_{2} \text { on } \\
\gamma-\mathrm{Al}_{2} \mathrm{O}_{3}\end{array}$ & $\begin{array}{l}\text { Refuse derived } \\
\text { fuel from } \\
\text { municipal solid } \\
\text { waste }\end{array}$ & $\begin{array}{c}723 \mathrm{~K}, 290 \mathrm{bar} \\
\rho_{\mathrm{H} 2 \mathrm{O}}=140 \mathrm{~kg} \mathrm{~m}^{-3}, \\
60 \mathrm{~min} \text { reaction time } \\
1.0 \mathrm{~g} \text { of feed, } 0.5 \mathrm{~g} \text { catalyst }\end{array}$ & $\begin{array}{c}93 \% \text { carbon gasification } \\
\text { efficiency } \\
36.9 \text { vol. } \% \mathrm{H}_{2} \\
23.2 \text { vol. } \% \mathrm{CH}_{4}\end{array}$ & $\begin{array}{l}\text { Yildirir, } \\
\text { Onwudili, } \\
\text { and } \\
\text { Williams } \\
2017 \text { [65] }\end{array}$ \\
\hline & $\begin{array}{c}\text { ZnO-doped } \\
\mathrm{Ni}-\mathrm{CaO} \\
5 \text { wt. \% } \mathrm{ZnO} \text { with } \\
5 \text { wt. } \% \mathrm{Ni}-\mathrm{CaO}\end{array}$ & $\begin{array}{l}\text { Biomass- } \\
\text { empty palm } \\
\text { fruit bunches } \\
\text { (EFBs) }\end{array}$ & $\begin{array}{c}653 \mathrm{~K}, \\
8 \mathrm{~min} \text { reaction time } 0.3 \mathrm{~g} \\
\text { feed, } 5 \mathrm{wt} . \% \text { catalyst, } \\
8 \mathrm{~mL} \text { deionized water }\end{array}$ & $\begin{array}{c}17.1 \% \text { conversion } \\
105.7 \mathrm{mmol} \mathrm{H}_{2} / \mathrm{mL} \text { feed }\end{array}$ & $\begin{array}{l}\text { Taufiq-Yap, } \\
\text { Sivasangar, } \\
\text { and } \\
\text { Surahim } \\
2019[67]\end{array}$ \\
\hline & $\mathrm{Co}_{2} \mathrm{O}_{3}$ & $\begin{array}{l}\text { Soda black } \\
\text { liquor }\end{array}$ & $\begin{array}{c}873 \mathrm{~K}, 240-260 \mathrm{bar}^{-} \\
\rho_{\mathrm{H} 2 \mathrm{O}}=67-74 \mathrm{~kg} \mathrm{~m}^{-3}, \\
30 \text { min reaction time } \\
\text { the mass ratio of catalyst } \\
\text { to feed } 1: 1\end{array}$ & $\begin{array}{l}71.05 \% \text { gasification } \\
\text { efficiency } \\
54.8 \% \mathrm{H}_{2} \text { fractions }\end{array}$ & $\begin{array}{l}\text { Cao et al. } \\
2020[128]\end{array}$ \\
\hline & $\mathrm{NiFe}_{2} \mathrm{O}_{4}$ & $\begin{array}{l}\text { Eucalyptus } \\
\text { wood chips }\end{array}$ & $\begin{array}{c}723 \mathrm{~K} \\
60 \mathrm{~min} \text { reaction time } 2.2 \mathrm{~g} \\
\text { feed, } 2.0 \mathrm{~g} \text { catalyst }\end{array}$ & $\begin{array}{l}95.49 \% \text { biomass } \\
\text { conversion } \\
25.05 \text { mol. } \% \mathrm{H}_{2}\end{array}$ & $\begin{array}{c}\text { Borges et al. } \\
2020 \text { [129] }\end{array}$ \\
\hline SCWO catalyst & $\mathrm{MnO}_{2}$ powder & $\begin{array}{l}\mathrm{NH}_{3}+\text { phenol } \\
\text { model solution }\end{array}$ & $\begin{array}{c}773 \mathrm{~K}, 247 \mathrm{bar} \\
\rho_{\mathrm{H} 2 \mathrm{O}}=88 \mathrm{~kg} \mathrm{~m}^{-3} \\
0.03 \mathrm{~min} \text { contact time } \\
\mathrm{C}_{\mathrm{NH} 3,0}=1.67 \mathrm{~mol} / \mathrm{m}^{3} \\
\mathrm{C}_{\mathrm{Ph}, 0}=0.107 \mathrm{~mol} / \mathrm{m}^{3} \\
\mathrm{C}_{\mathrm{O} 2,0}=11.0 \mathrm{~mol} / \mathrm{m}^{3}\end{array}$ & $\begin{array}{l}>90 \% \text { NH3 conversion } \\
>90 \% \text { TOC conversion }\end{array}$ & $\begin{array}{l}\text { Oshima, } \\
\text { Inaba, and } \\
\text { Koda 2001 } \\
\text { [98] }\end{array}$ \\
\hline
\end{tabular}


Table 2. Cont.

\begin{tabular}{|c|c|c|c|c|c|}
\hline $\begin{array}{l}\text { SCW Process } \\
\text { Functionality }\end{array}$ & Metal Oxide & Feed & $\begin{array}{l}\text { Optimal Reaction } \\
\text { Conditions }^{1}\end{array}$ & $\begin{array}{l}\text { Efficiency and } \\
\text { Selectivity }\end{array}$ & Reference \\
\hline & $\mathrm{Mn}_{2} \mathrm{O}_{3} / \mathrm{Ti}-\mathrm{Al}$ & $\begin{array}{l}\text { p-nitrophenol } \\
\text { wastewater }\end{array}$ & $\begin{array}{c}693 \mathrm{~K}, 260 \mathrm{bar}, \\
\rho_{\mathrm{H} 2 \mathrm{O}}=143 \mathrm{~kg} \mathrm{~m}^{-3}, \\
0.1 \mathrm{~min} \text { residence time } \\
1000 \mathrm{mg} / \mathrm{L} \text { feed } \\
\text { concentration, } 6.5 \mathrm{~g} \text { of } \\
\text { catalyst }\end{array}$ & $99 \%$ conversion & $\begin{array}{l}\text { Dong et al. } \\
2015 \text { [130] }\end{array}$ \\
\hline \multirow{2}{*}{$\begin{array}{l}\text { SCW S-removal } \\
\text { sorbent }\end{array}$} & $\mathrm{ZnO}$ & $\begin{array}{l}\text { refractory } \\
\text { sulfur } \\
\text { compound } \\
\text { from crude oil }\end{array}$ & $\begin{array}{c}673 \mathrm{~K}, 250 \mathrm{bar}^{\prime} \\
\rho_{\mathrm{H} 2 \mathrm{O}}=167 \mathrm{~kg} \mathrm{~m}^{-3}, \\
60 \mathrm{~min} \text { reaction time } \\
1: 1 \text { wt./wt. of oil/water, } \\
0.35 \mathrm{~g} \text { catalyst }\end{array}$ & $\begin{array}{l}25 \% \text { efficiency of sulfur } \\
\text { removal }\end{array}$ & $\begin{array}{l}\text { Ates et al. } \\
2014[131]\end{array}$ \\
\hline & $\begin{array}{c}\mathrm{ZnO} \\
\text { Katalco }\end{array}$ & $\begin{array}{l}\text { Chlorella } \\
\text { vulgaris }\end{array}$ & $\begin{array}{c}673 \mathrm{~K}, 280 \mathrm{bar}^{\prime} \\
\rho_{\mathrm{H} 2 \mathrm{O}}=261 \mathrm{~kg} \mathrm{~m}^{-3} \\
6000 \text { min stream time } \\
31 \mathrm{~g} \mathrm{~S} \text { in feed }\end{array}$ & $\begin{array}{l}8 \% \text { efficiency of sulfur } \\
\text { removal }\end{array}$ & $\begin{array}{l}\text { Peng et al. } \\
2017 \text { [132] }\end{array}$ \\
\hline
\end{tabular}

${ }^{1}$ Water density values from NIST database at https:/ / webbook.nist.gov/ chemistry/fluid/ (accessed on 28 October 2021).

Various transition metal oxides have been tested for the gasification of biomass and biomass related model compounds, and for gas production from industrial and municipal wastes under different SCW conditions [67,72,128,133].

At $773 \mathrm{~K}, 314$ bar for $60 \mathrm{~min}$, the gasification of sunflower stalk and corncob as lignocellulosic feedstock and leather waste as a proteinous biomass was highly efficient when using red mud, an Fe-oxide-containing residue from Al production by the Bayer process, as the catalyst. This catalyst exhibits good selectivity for $\mathrm{H}_{2}$ and $\mathrm{CO}_{2}$ production. It is notable that a very small amount of coke was generated when gasification was started from corncob and leather waste. This study proved that iron-based materials exhibit catalytic activity for the production of $\mathrm{H}_{2}$ from biomass [115].

Gasification of soda black liquor was tested in SCW at $873 \mathrm{~K}$ and pressures ranging from 240 to 260 bar, over various transition metal oxides, such as $\mathrm{V}_{2} \mathrm{O}_{5}, \mathrm{Cr}_{2} \mathrm{O}_{3}, \mathrm{MnO}_{2}$, $\mathrm{Fe}_{2} \mathrm{O}_{3}, \mathrm{Co}_{2} \mathrm{O}_{3}, \mathrm{CuO}, \mathrm{ZnO}, \mathrm{ZrO}_{2}, \mathrm{MoO}_{3}, \mathrm{WO}_{3}, \mathrm{TiO}_{2}$, and $\mathrm{Fe}_{3} \mathrm{O}_{4}$. The highest gasification efficiency was achieved when $\mathrm{V}_{2} \mathrm{O}_{5}$ was used as catalyst, however with low $\mathrm{H}_{2}$ selectivity. The highest $\mathrm{H}_{2}$ fraction in the gas product was obtained when $\mathrm{ZnO}$ and $\mathrm{Co}_{2} \mathrm{O}_{3}$ were used as catalysts in the gasification reaction [128]. When the same metal oxides were tested for the gasification of glucose model compound, all the metal oxides improved the $\mathrm{CH}_{4}$ fractions except $\mathrm{V}_{2} \mathrm{O}_{5}, \mathrm{CuO}$, and $\mathrm{ZrO}_{2}$ [72]. Under the investigated processing conditions, the least stable catalysts were found to be $\mathrm{CuO}$ and $\mathrm{Fe}_{2} \mathrm{O}_{3}$ which reduced to metal or lower-valence metal oxides $\left(\mathrm{Cu}, \mathrm{Cu}_{2} \mathrm{O}\right.$, or $\left.\mathrm{FeO}\right)$ without catalytic activity [128].

A $\mathrm{ZnO}$-doped $\mathrm{Ni}-\mathrm{CaO}$ catalyst, specifically at $5 \mathrm{wt}$. \% $\mathrm{ZnO}$ with a $\mathrm{Ni}$ loading of 5 wt. \% on $\mathrm{CaO}$ showed high feedstock conversion and $\mathrm{H}_{2}$ production by empty palm fruit bunch biomass gasification at $653 \mathrm{~K}$ and $8 \mathrm{~min}$ reaction time. Hydrogen made up the largest proportion in the resulting gas which consisted mainly of $\mathrm{H}_{2}$ and $\mathrm{CO}_{2}, \mathrm{CH}_{4}$, and CO. It was suggested that the formation of an $\mathrm{Ni}_{0.8} \mathrm{Zn}_{0 \cdot 2} \mathrm{O}$ phase from the strong interaction between the dopants actively promoted the water-gas shift reaction [67].

An $\mathrm{RuO}_{2}$ catalyst supported on $\gamma-\mathrm{Al}_{2} \mathrm{O}_{3}$ was used for the gasification of refuse-derived fuel from municipal solid waste at $773 \mathrm{~K}$, and 290 bar. With an Ru loading of $20 \mathrm{wt}$. \% on the support, the attained carbon gasification efficiency was $93 \%$ after $60 \mathrm{~min}$ residence time. Although the largest fraction in the gas product was $\mathrm{CO}_{2}$, almost $37 \% \mathrm{H}_{2}$ and $23 \%$ of $\mathrm{CH}_{4}$ were obtained under optimal reaction conditions. However, the $\mathrm{CO}_{2}$ fraction decreased with increasing reaction time [65].

Rare-earth metal oxides, such as $\mathrm{CeO}_{2}$, showed little catalytic activity within the gasification reaction of glucose at $873 \mathrm{~K}, 250$ bar, and 10 min reaction time in a quartz capillary reactor [72] and the same for the gasification of soda black liquor at $873 \mathrm{~K}$ and 
240-260 bar [128]. Cubic $\mathrm{CeO}_{2}$ nanocatalyst was tested for the gasification of black liquor in sub- and supercritical conditions at $623 \mathrm{~K}$ and $723 \mathrm{~K}, 250$ bar in a batch reactor for $15 \mathrm{~min}$ and $60 \mathrm{~min}$. The carbon conversion of black liquor at sub and supercritical conditions improved over the catalyst. The selectivity for $\mathrm{H}_{2}$ formation in SCW \& 60 min was not high, as the amount of $\mathrm{H}_{2}$ was only slightly affected by the catalyst compared to the non-catalytic conditions. It was shown that, at longer reaction times and in sub- rather than supercritical conditions of water, the $\mathrm{CeO}_{2}$ catalyst improves the water gas shift reaction which increases the $\mathrm{H}_{2}$ yield. The amount of coke produced without the catalyst was lower than that with the catalyst [127].

The activity of $\mathrm{NiFe}_{2} \mathrm{O}_{4}$ spinel-type catalyst was examined, as a function of temperature, at 673,723 , and $773 \mathrm{~K}$ for $60 \mathrm{~min}$ in the gasification of eucalyptus wood chips. A conversion of $95 \%$ compared to $73 \%$ in the non-catalytic process was attained, with the highest $\mathrm{H}_{2}$ fraction formed at $723 \mathrm{~K}$. Tests of recyclability under the operating conditions using recovered and recalcined catalysts indicated coke formation and significant deactivation after a third reaction cycle, leading to reduced biomass conversion and $\mathrm{H}_{2}$ yield [129].

Figure 4 shows a selection of optimal operation conditions in terms of temperature and pressure at which various catalysts performed best in SCWG processes of different waste feedstocks. We selected data from Tables 1 and 2 and present them in Figure 4 relative to the PB-Widom line as determined in [19]. The first thing to observe in Figure 4 is that all catalysts perform well at pressures below 350 bar. Secondly, it is interesting to note that Ru-based catalysts used for SCWG of biomass to obtain $\mathrm{CH}_{4}$ as the main gas product have the best performance at conditions close to the PB-Widom line. The other selected catalysts show high catalytic activity in the GL supercritical region of water. However, Ni-based catalysts seem to show the highest selectivity for $\mathrm{H}_{2}$ formation at relatively higher temperatures than bimetallic catalysts or metal oxides.

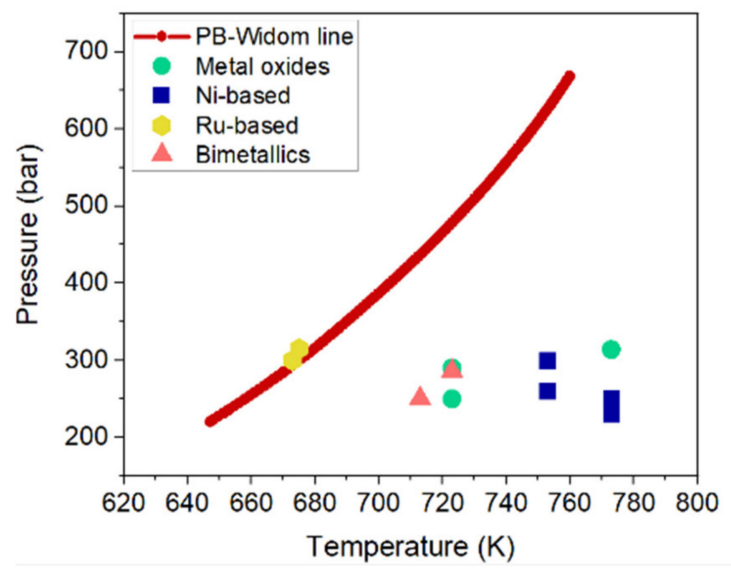

Figure 4. Selected optimal pressure-temperature conditions for SCWG processes over various catalysts; a selection taken from Tables 1 and 2 is presented relative to the PB-Widom line [19].

Screening the selected literature, we found that catalytic SCWO is not a common practice, especially in recent years. This could be because the SCW by itself is active in the oxidation of most organic compounds, which activity is enhanced by the addition of the oxidative agent. Moreover, in some cases, it was reported that the SCW holds back, for instance, the conversion of $\mathrm{NH}_{3}$ into $\mathrm{N}_{2}$ and $\mathrm{N}_{2} \mathrm{O}$ products, due to adsorption inhibition by water during the oxidation reaction over bulk $\mathrm{MnO}_{2}$ catalyst [98].

Most of the experiments on the oxidative catalysis in SCW are performed starting from model solutions and using $\mathrm{MnO}_{2}$-based catalysts $[25,98,108,130,134]$.

The commercial catalyst CARULITE 150 with a composition of $45-60 \% \mathrm{MnO}_{2}$ and $1-3 \% \mathrm{CuO}$ on amorphous $\mathrm{Al}_{2} \mathrm{O}_{3}$ support was used for the oxidation of phenol under various operation conditions. Using this catalyst, much higher phenol conversion and 
selectivity to $\mathrm{CO}_{2}$ formation was achieved compared to the non-catalytic process at $658 \mathrm{~K}$ and 253 bar in presence of $\mathrm{O}_{2}$. The phenol conversion was always higher than $70 \%$, achievable in $2 \mathrm{~s}$ of space time, defined here as the ratio between the catalyst mass to the fluid flow rate. In aged conditions, the catalyst maintained high activity even after several days of continuous operation [108]. The activity for the phenol oxidation of bulk $\mathrm{TiO}_{2}$ and $\mathrm{MnO}_{2}$ versus that of commercial CARULITE was compared at $654 \mathrm{~K}$ and 253 bar. Only over the CARULITE catalyst did the conversion of phenol reach $100 \%$, after less than $1 \mathrm{~s}$ of space time. While, the bulk $\mathrm{TiO}_{2}$ and $\mathrm{MnO}_{2}$ provided $\mathrm{CO}_{2}$ selectivity similar to the non-catalytic process, i.e., no more than $40 \%$, the commercial catalyst exhibited high selectivity to $\mathrm{CO}_{2}$ formation, approaching $60 \%$ when the phenol oxidation was complete [25].

$\mathrm{MnO}_{2}$, prepared by compression molding with nitrocellulose as pore forming agent and calcinated afterwards, it was catalytically tested for the oxidation of nitrobenzene at $738 \mathrm{~K}, 280$ bar. Under these conditions, the degradation efficiency was higher than $99 \%$ even after $18 \mathrm{~h}$ on stream, showing the stability of the catalyst in SCW [134]. Using bulk $\mathrm{MnO}_{2}$ catalyst and $\mathrm{H}_{2} \mathrm{O}_{2}$ as oxidant, experiments on the oxidation of diluted coke-works waste, containing manly $\mathrm{NH}_{3}$ and phenol, were performed at $773 \mathrm{~K}$ and $247 \mathrm{bar}$. A high total organic carbon removal efficiency was achieved, and a high conversion to $\mathrm{N}_{2}$ and $\mathrm{N}_{2} \mathrm{O}$, as minor product, with a selectivity of up to $90 \%$ [98].

$\mathrm{Mn}_{2} \mathrm{O}_{3}$ supported on Ti-Al oxide composite, prepared by incipient wet impregnation using $\mathrm{Mg}\left(\mathrm{NO}_{3}\right)_{2}$, has been tested as a catalyst for oxidation of p-nitrophenol-containing wastewater, using compressed air and $\mathrm{H}_{2} \mathrm{O}_{2}$ as oxidants, in the temperature interval from $658 \mathrm{~K}$ to $718 \mathrm{~K}$, at 260 bar and $1-2 \mathrm{~s}$ reaction time. Compared to the activity of unsupported $\mathrm{MnO}_{2}$ and $\mathrm{Mn}_{2} \mathrm{O}_{3}$, the supported catalysts showed the highest removal efficiency, close to $100 \%$, at the optimal temperature for the catalytic process of $693 \mathrm{~K}$ [130].

Transition metal oxide materials were used also for sulfur removal from SCW streams. For instance, $\mathrm{ZnO}$ and $\mathrm{MoO}_{3}$ materials promoted sulfur removal during the decomposition in SCW of the aliphatic sulfide compounds contained in crude oil. The SCW desulfurization performance of such compounds increased from 3 to $25 \%$ when using $\mathrm{ZnO}$ as the sorbent material at $673 \mathrm{~K}$ and 250 bar. In fact, the SCW alone removed $6-7 \%$ of the sulfur present in the investigated crude oil [131]. The sorption mechanism is related to the sulfidation reaction based on the ability of metal oxides to replace the $\mathrm{O}$ with $\mathrm{S}$. Commercial $\mathrm{ZnO}$ exhibited good sulfur adsorption performance under SCW conditions in the catalytic gasification of microalgae at $673 \mathrm{~K}$ and 280 bar. The adsorbent bed, placed before the catalytic bed, was used to avoid the poisoning by S species of the Ru catalyst [132].

\subsection{Supporting Materials and Promoters}

The supporting materials employed in SCW processes, should have chemical stability, high surface area and to allow good dispersion of the catalyst particles onto their surface, while offering easy accessibility to the active sites. Two main types of catalyst supports are used in processes undergoing in SCW conditions. These are the metal-oxides, such as $\mathrm{Al}_{2} \mathrm{O}_{3}, \mathrm{TiO}_{2}, \mathrm{ZrO}_{2}, \mathrm{CeO}_{2}, \mathrm{ZrO}_{2}$, and $\mathrm{MgO}[52,68,135]$ and carbon-based materials such as activated carbon (AC), CNTs, and functionalized CNTs [116]. The former are stable compounds, chemically inert, and exhibit strong mechanic properties. The latter materials are attractive as supports for catalytic processes due to their large specific surface areas of $\sim 800-1500 \mathrm{~m}^{2} \mathrm{~g}^{-1}$ [31], high heat conductivity, porosity, and stability with regard to morphological, physicochemical and thermal properties [116]. Still, the activated carbon's relative instability in multiple utilizations may limit the practical applications of these supporting materials compared to the metal oxide-supported catalyst [52].

Various metal oxides, such as $\mathrm{ZrO}_{2}, \mathrm{CeO}_{2}-\mathrm{ZrO}_{2}, \mathrm{Al}_{2} \mathrm{O}_{3}, \mathrm{MgO}-\mathrm{Al}_{2} \mathrm{O}_{3}$ have been employed as supporting materials for the synthesis of Ni-based catalysts at $773 \mathrm{~K}$ and 230 bar [135]. The highest activity in the SCWG of glycerol was exhibited by the catalyst supported on $\mathrm{MgO}-\mathrm{Al}_{2} \mathrm{O}_{3}$. This is due to the fact that the support allows high dispersion of the metallic component. Moreover, spinel-type oxides, e.g., $\mathrm{NiAl}_{2} \mathrm{O}_{4}$ and $\mathrm{MgAl}_{2} \mathrm{O}_{4}$ are formed with catalytic activity. In addition, the $\mathrm{MgO}-\mathrm{Al}_{2} \mathrm{O}_{3}$ support has an extremely stable 
mesoporous structure in long-term SCW operations, an excellent coke resistance, and it is also easy to regenerate. The catalyst prepared on $\mathrm{CeO}_{2}-\mathrm{ZrO}_{2}$ shows the lowest performance in the SCWG of glycerol due to low dispersion of active-Ni [135]. In another recent study on supported Ni catalysts for SCWG reaction it was found that the contribution of metal oxides as supporting materials for the formation of $\mathrm{H}_{2}$ gas product decreased in the following order: $\mathrm{ZrO}_{2}>\mathrm{Al}_{2} \mathrm{O}_{3}>\mathrm{SiO}_{2}>\mathrm{Al}_{2} \mathrm{O}_{3}-\mathrm{SiO}_{2}$. The study pointed out the importance of high surface area of catalysts during SCWG as well as the active metal dispersion within the support. Moreover, coke formation, and metal-support interactions played important role in the catalysts activity and selectivity [68]. Simple metal oxides were employed as supporting materials also for the Ru-based catalysts tested in the SCWG and it was found that the carbon gasification efficiency decreased in the following order: $\mathrm{TiO}_{2}>\mathrm{CeO}_{2}>$ $\mathrm{ZrO}_{2}>\mathrm{Al}_{2} \mathrm{O}_{3}>\mathrm{MgO}$ [52].

The effect of carbon-based supports was evaluated in the SCWG of sugarcane bagasse over a Ru catalyst, and it was found that the support pore structure is modified due to blocking of the pores by the formation of carbonaceous products during the gasification process. The activity of the metallic catalyst drastically dropped during repetitive SCWG processes, therefore only fresh catalyst could be used [50]. Graphite and charcoal supporting materials for Ru catalysts employed in the SCWG of lignin were compared and it was found that the pore structure of the carbon supports played a crucial role in the catalytic activity [136]. When using graphite supported ruthenium catalysts, superior gasification yield was recorded, although the graphite has a six-times-smaller micropore volume than charcoal. The explanation is related to partially hydrolyzed lignin molecules that, due to their volume, were not able to reach the catalyst particles located within charcoal micropores [136]. The CNTs used as supports for Ni-based catalysts were found to be efficient in SCWG processes [116,135]. It was found that Ni/CNT catalyst prepared in SCW exhibited higher activity and increased stability compared to Ni/AC catalyst [135]. Moreover, functionalized CNTs, with increased surface area, proved to be efficient in obtaining $\mathrm{H}_{2}$-rich gas from light gas oil and heavy gas oil by SCWG over an Ni-based catalyst. The support's high porosity facilitated the easy access of the waste/feedstock to the active sites of the catalyst [116].

In catalysis, promoters are substances that, added in small amounts to a given catalyst during preparation, work to improve its properties such as the activity (facilitate the desired reaction) and selectivity (suppress unwanted processes) as in the case of chemical promoters, or to improve stability (as in preventing sintering, physical promoters). By themselves, promoters have little or no activity. The effects of adding promoters such as $\mathrm{Na}, \mathrm{K}$, and $\mathrm{Ce}$ to Ni-supported catalysts on $\mathrm{ZrO}_{2}$ and $\mathrm{Al}_{2} \mathrm{O}_{3}$ catalysts were recently evaluated and the study showed an increased $\mathrm{Ni}$ dispersion which improved $\mathrm{H}_{2}$ yields during SCWG [68]. It was found that $\mathrm{CeO}_{2}$ can be used as promoter for coke removal from $\mathrm{Ni}$ catalyst surfaces by oxidizing the surface-deposited char due to its oxygen mobility and oxygen retaining ability [137]. Moreover, La promoter added to $\mathrm{Al}_{2} \mathrm{O}_{3}$-supported nickel catalyst diminished the carbon deposition and maintained catalyst activity over multiple cycles of food waste gasification experiments at $753 \mathrm{~K}$ [122].

\section{Conclusions}

In conclusion, we carefully screened the literature from recent decades reporting results related to waste treatment by SCWG and/or SCWO. We focused on functional materials, such as metal-based catalysts efficient for the gasification processes in SCW, and metal oxides with catalytic and sorption activity in both SCWG and SCWO. After processing the high amount of information on SCW processes starting from wastes and ending with energy-relevant products, we learned the following lessons:

- To overcome the issues related to the economic challenges coming from high operation costs of SCW systems and the technological problems caused by corrosion and salt deposition, it is necessary to optimize the operation parameters for enhanced energy efficiency and savings. 
- Managing to finely tune the properties of SCW is the key towards the operating parameters optimization. For this, fundamental knowledge on the supercritical state of water is essential. During isobaric heating in the supercritical region of a fluid, the $\mathrm{PB}$ phenomenon occurs, which is reflected in the fluid structural changes from LL to GL phases, across the PB-Widom line. The water properties change drastically at this phase transition, and reaction mechanisms in LL water are different to those in the GL phase.

- Heterogeneous catalysis in SCW allows efficient treatment of various types of waste at relatively low temperatures, mostly in the GL region of water, with enhanced selectivity for the formation of desired products in a short time.

- Among metal functional materials for SCWG processes, the Ni-based catalysts are mostly active at temperatures higher than $773 \mathrm{~K}$, enhancing the rates of reforming or water-gas shift reactions. Therefore, gasification over these catalysts results in the formation of an $\mathrm{H}_{2}$-rich gas product. Noble metals, such as Ru-based catalysts, are efficient at temperatures as low as $673 \mathrm{~K}$ and have high catalytic activity for reactions with $\mathrm{CH}_{4}$ as main gas product, especially when the feedstock is based on microalgae biomass.

- Metal oxides are used as functional materials in both SCWG and SCWO treatment of wastes feedstock. Transition metal oxides mostly show catalytic activity in the gasification reactions of various types of wastes at temperatures of $763 \mathrm{~K}$ on average. For the SCWO only manganese-based oxides exhibit catalytic activity towards complete conversion of organic matter, obtained in seconds at temperatures not higher than $800 \mathrm{~K}$. ZnO shows efficiency in the adsorption of sulfur from the SCW streams, and thus the poisoning and deactivation of catalysts is avoided.

- Supporting materials based on metal oxides prove to be more suitable for long term SCW operations, and more resistant to coke depositions than the carbon materials used as supports for catalysts, although the later provide higher surface area, and enhanced dispersibility of the catalyst particles can be achieved.

- Promoters such as rare earth-based materials increase the stability of catalysts under SCW conditions by reducing the coke/char deposition on the catalyst surface and thus avoiding the deactivation.

The information on waste-to-energy processes in SCW, selected and discussed here in correlation with fundamental water science, aims to be a practical guide for the selection of materials stable and functional under supercritical conditions.

Author Contributions: Conceptualization, F.M.; investigation, I.P., E.E.T., G.S.S. and F.T.; writingoriginal draft preparation, F.M.; writing-review and editing, F.M., I.P., E.E.T., F.T. and S.T.; visualization, F.M. and C.H.; supervision, F.M. and S.T. All authors have read and agreed to the published version of the manuscript.

Funding: This research was funded by Romanian Ministry of Research, Innovation and Digitization, CNCS/CCCDI-UEFISCDI, grant number PN-III-P4-ID-PCE-2020-1241, within PNCDI III.

Institutional Review Board Statement: Not applicable.

Informed Consent Statement: Not applicable.

Acknowledgments: The work with the setup working under supercritical water conditions at "Ilie Murgulescu" Institute of Physical Chemistry was possible due to the Research Collaboration Agreement between the Romanian Institution and the Laboratory for Bioenergy and Catalysis (LBK), ENE Division, Paul Scherrer Institute, 5232 Villigen PSI, Switzerland, group of Christian Ludwig, who is gratefully acknowledged. F.M. thanks Daniel T. Banuti from the University of New Mexico, USA, for providing data for the PB-Widom line presented in Figures 1 and 4, and Pierre Boillat for processing the neutron images showed in Figure 2. The authors thank to Cristian Contescu from ORNL, USA, for donation of carbon-based materials used for experiments at supercritical water conditions. 
Conflicts of Interest: The authors declare no conflict of interest. The funders had no role in the design of the study; in the collection, analyses, or interpretation of data; in the writing of the manuscript, or in the decision to publish the results.

\section{References}

1. Stucki, S.; Ludwig, C. Municipal Solid Waste Management; Ludwig, C., Hellweg, S., Stucki, S., Eds.; Springer: Berlin/Heidelberg, Germany, 2003; ISBN 978-3-642-62898-6.

2. Guerrero, L.A.; Maas, G.; Hogland, W. Solid waste management challenges for cities in developing countries. Waste Manag. 2013, 33, 220-232. [CrossRef]

3. Office of the European Union Horizon Europe Strategic plan 2021-2024. Available online: https:/ / op.europa.eu/en/publicationdetail/- / publication/3c6ffd74-8ac3-11eb-b85c-01aa75ed71a1/language-en/format-PDF/source-search (accessed on 27 September 2021).

4. European Environment Agency Waste Management. Available online: https://www.eea.europa.eu/themes/waste/wastemanagement (accessed on 9 September 2021).

5. Căta, A.; Miclău, M.; Ienaşcu, I.; Ursu, D.; Tănasie, C.; Ştefenuţa, M.N. Chemical recycling of Polyethylene Terephthalate (PET) waste using Sub- and supercritical water. Rev. Roum. Chim. 2015, 60, 579-585.

6. Čolnik, M.; Knez, Ž.; Škerget, M. Sub- and supercritical water for chemical recycling of polyethylene terephthalate waste. Chem. Eng. Sci. 2021, 233. [CrossRef]

7. Liu, Y.; Fan, C.; Zhang, H.; Zou, J.; Zhou, F.; Jin, H. The resource utilization of ABS plastic waste with subcritical and supercritical water treatment. Int. J. Hydrogen Energy 2019, 44, 15758-15765. [CrossRef]

8. Goto, M.; Sasaki, M.; Hirose, T. Reactions of polymers in supercritical fluids for chemical recycling of waste plastics. J. Mater. Sci. 2006, 41, 1509-1515. [CrossRef]

9. Queiroz, A.; Pedroso, G.B.; Kuriyama, S.N.; Fidalgo-Neto, A.A. Subcritical and supercritical water for chemical recycling of plastic waste. Curr. Opin. Green Sustain. Chem. 2020, 25, 100364. [CrossRef]

10. Goto, M. Supercritical water process for the chemical recycling of waste plastics. AIP Conf. Proc. 2010, 1251, 169-172. [CrossRef]

11. Li, K.; Xu, Z. A review of current progress of supercritical fluid technologies for e-waste treatment. J. Clean. Prod. 2019, 227, 794-809. [CrossRef]

12. Xiu, F.R.; Qi, Y.; Zhang, F.S. Recovery of metals from waste printed circuit boards by supercritical water pre-treatment combined with acid leaching process. Waste Manag. 2013, 33, 1251-1257. [CrossRef]

13. Hong, G.T.; Fowler, P.K.; Killilea, W.R.; Swallow, K.C. Supercritical water oxidation: Treatment of human waste and system configuration tradeoff study; SAE Technical Paper, 1987; SAE International: Warrendale, PA, USA, 1987. [CrossRef]

14. Barner, H.E.; Huang, C.Y.; Johnson, T.; Jacobs, G.; Martch, M.A.; Killilea, W.R. Supercritical water oxidation: An emerging technology. J. Hazard. Mater. 1992, 31, 1-17. [CrossRef]

15. Zhang, D.; Luther, A.K.; Clauwaert, P.; Ciccioli, P.; Ronsse, F. Assessment of carbon recovery from solid organic wastes by supercritical water oxidation for a regenerative life support system. Environ. Sci. Pollut. Res. 2020, 27, 8260-8270. [CrossRef] [PubMed]

16. Boukis, N.; Katharina Stoll, I. Gasification of biomass in supercritical water, challenges for the process design-lessons learned from the operation experience of the first dedicated pilot plant. Processes 2021, 9, 455. [CrossRef]

17. Haiduc, A.G.; Brandenberger, M.; Suquet, S.; Vogel, F.; Bernier-Latmani, R.; Ludwig, C. SunCHem: An integrated process for the hydrothermal production of methane from microalgae and $\mathrm{CO}_{2}$ mitigation. J. Appl. Phycol. 2009, 21, 529-541. [CrossRef]

18. Bagnoud-Velásquez, M.; Brandenberger, M.; Vogel, F.; Ludwig, C. Continuous catalytic hydrothermal gasification of algal biomass and case study on toxicity of aluminum as a step toward effluents recycling. Catal. Today 2014, 223, 35-43. [CrossRef]

19. Maxim, F.; Karalis, K.; Boillat, P.; Banuti, D.T.; Marquez Damian, J.I.; Niceno, B.; Ludwig, C. Thermodynamics and Dynamics of Supercritical Water Pseudo-Boiling. Adv. Sci. 2021, 8, 2002312. [CrossRef]

20. Karalis, K.; Ludwig, C.; Niceno, B. Supercritical water anomalies in the vicinity of the Widom line. Sci. Rep. $2019,9,15731$. [CrossRef]

21. Peterson, A.A.; Vogel, F.; Lachance, R.P.; Fröling, M.; Antal, M.J., Jr.; Tester, J.W. Thermochemical biofuel production in hydrothermal media: A review of sub- and supercritical water technologies. Energy Environ. Sci. 2008, 1, 32. [CrossRef]

22. Vadillo, V.; Sánchez-Oneto, J.; Portela, J.R.; Martínez de la Ossa, E.J. Supercritical Water Oxidation. In Advanced Oxidation Processes for Waste Water Treatment; Elsevier: Amsterdam, The Netherlands, 2018; pp. 333-358; ISBN 9780128105252.

23. Ortiz, F.J.G.; Campanario, F.J.; Aguilera, P.G.; Ollero, P. Hydrogen production from supercritical water reforming of glycerol over $\mathrm{Ni} / \mathrm{Al}_{2} \mathrm{O}_{3}-\mathrm{SiO}_{2}$ catalyst. ENERGY 2015, 84, 634-642. [CrossRef]

24. Gutiérrez Ortiz, F.J.; Serrera, A.; Galera, S.; Ollero, P. Experimental study of the supercritical water reforming of glycerol without the addition of a catalyst. Energy 2013, 56, 193-206. [CrossRef]

25. Savage, P.E. Heterogeneous catalysis in supercritical water. Catal. Today 2000, 62, 167-173. [CrossRef]

26. Okolie, J.A.; Rana, R.; Nanda, S.; Dalai, A.K.; Kozinski, J.A. Supercritical water gasification of biomass: A state-of-the-art review of process parameters, reaction mechanisms and catalysis. Sustain. Energy Fuels 2019, 3, 578-598. [CrossRef]

27. Shirai, M.; Yamaguchi, A.; Hiyoshi, N.; Sato, O. Effect of Sulfur on Gasification of Lignin over Supported Metal Catalysts in Supercritical Water. Sci. Technol. 2008, 930, 2008. 
28. Peng, G.; Ludwig, C.; Vogel, F. Catalytic supercritical water gasification: Interaction of sulfur with $\mathrm{ZnO}$ and the ruthenium catalyst. Appl. Catal. B Environ. 2017, 202, 262-268. [CrossRef]

29. Elliott, D.C. Catalytic hydrothermal gasification of biomass. Biofuels Bioprod. Biorefining 2008, 2, 254-265. [CrossRef]

30. Guo, Y.; Wang, S.Z.; Xu, D.H.; Gong, Y.M.; Ma, H.H.; Tang, X.Y. Review of catalytic supercritical water gasification for hydrogen production from biomass. Renew. Sustain. Energy Rev. 2010, 14, 334-343. [CrossRef]

31. Azadi, P.; Farnood, R. Review of heterogeneous catalysts for sub- and supercritical water gasification of biomass and wastes. Int. J. Hydrogen Energy 2011, 36, 9529-9541. [CrossRef]

32. Reddy, S.N.; Nanda, S.; Dalai, A.K.; Kozinski, J.A. Supercritical water gasification of biomass for hydrogen production. Int. J. Hydrogen Energy 2014, 39, 6912-6926. [CrossRef]

33. Rodriguez Correa, C.; Kruse, A. Supercritical water gasification of biomass for hydrogen production - Review. J. Supercrit. Fluids 2018, 133, 573-590. [CrossRef]

34. Savage, P.E.; Dunn, J.B.; Yu, J. Recent advances in catalytic oxidation in supercritical water. Combust. Sci. Technol. 2006, 178, 443-465. [CrossRef]

35. Savage, P.E. A perspective on catalysis in sub- and supercritical water. J. Supercrit. Fluids 2009, 47, 407-414. [CrossRef]

36. Atkins, P.; de Paula, J. Physical Chemistry, 8th ed; W. H. Freeman and Company: New York, NY, USA, 2006.

37. Simeoni, G.G.; Bryk, T.; Gorelli, F.A.; Krisch, M.; Ruocco, G.; Santoro, M.; Scopigno, T. The Widom line as the crossover between liquid-like and gas-like behaviour in supercritical fluids. Nat. Phys. 2010, 6, 503-507. [CrossRef]

38. Brazhkin, V.V.; Lyapin, A.G.; Ryzhov, V.N.; Trachenko, K.; Fomin, Y.D.; Tsiok, E.N. Where is the supercritical fluid on the phase diagram? Physics-Uspekhi 2012, 55, 1061-1079. [CrossRef]

39. McMillan, P.F.; Stanley, H.E. Fluid phases: Going supercritical. Nat. Phys. 2010, 6, 479-480. [CrossRef]

40. Gallo, P.; Corradini, D.; Rovere, M. Widom line and dynamical crossovers as routes to understand supercritical water. Nat. Commun. 2014, 5, 5806. [CrossRef] [PubMed]

41. Bolmatov, D.; Zav'yalov, D.; Gao, M.; Zhernenkov, M. Structural Evolution of Supercritical $\mathrm{CO}_{2}$ across the Frenkel Line. J. Phys. Chem. Lett. 2014, 5, 2785-2790. [CrossRef] [PubMed]

42. Nishikawa, K.; Tanaka, I. Correlation lengths and density fluctuations in supercritical states of carbon dioxide. Chem. Phys. Lett. 1995, 244, 149-152. [CrossRef]

43. Nishikawa, K.; Kusano, K.; Arai, A.A.; Morita, T. Density fluctuation of a van der Waals fluid in supercritical state. J. Chem. Phys. 2003, 118, 1341-1346. [CrossRef]

44. Banuti, D.T.; Raju, M.; Ihme, M. Between supercritical liquids and gases - Reconciling dynamic and thermodynamic state transitions. J. Supercrit. Fluids 2020, 165, 104895. [CrossRef]

45. Banuti, D.T. Crossing the Widom-line - Supercritical pseudo-boiling. J. Supercrit. Fluids 2015, 98, 12-16. [CrossRef]

46. Banuti, D.T.; Raju, M.; Ihme, M. Similarity law for Widom lines and coexistence lines. Phys. Rev. E 2017, 95, 052120. [CrossRef] [PubMed]

47. Wagner, W.; Pruß, A. The IAPWS formulation 1995 for the thermodynamic properties of ordinary water substance for general and scientific use. J. Phys. Chem. Ref. Data 2002, 31, 387-535. [CrossRef]

48. Maxim, F.; Contescu, C.; Boillat, P.; Niceno, B.; Karalis, K.; Testino, A.; Ludwig, C. Visualization of supercritical water pseudoboiling at Widom line crossover. Nat. Commun. 2019, 10, 4114. [CrossRef] [PubMed]

49. Osada, M.; Sato, O.; Watanabe, M.; Arai, K.; Shirai, M. Water Density Effect on Lignin Gasification over Supported Noble Metal Catalysts in Supercritical Water. Energy Fuels 2006, 20, 930-935. [CrossRef]

50. Osada, M.; Yamaguchi, A.; Hiyoshi, N.; Sato, O.; Shirai, M. Gasification of sugarcane bagasse over supported ruthenium catalysts in supercritical water. Energy Fuels 2012, 26, 3179-3186. [CrossRef]

51. Sato, T.; Osada, M.; Watanabe, M.; Shirai, M.; Arai, K. Gasification of Alkylphenols with Supported Noble Metal Catalysts in Supercritical Water. Ind. Eng. Chem. Res. 2003, 42, 4277-4282. [CrossRef]

52. Shan, Y.Q.; Yin, L.X.; Djandja, O.S.; Wang, Z.C.; Duan, P.G. Supercritical water gasification of waste water produced from hydrothermal liquefaction of microalgae over Ru catalyst for production of $\mathrm{H}_{2}$ rich gas fuel. Fuel 2021, 292, 120288. [CrossRef]

53. Akizuki, M.; Oshima, Y. Acid catalytic properties of $\mathrm{TiO}_{2}, \mathrm{Nb}_{2} \mathrm{O}_{5}$, and $\mathrm{NbOX} / \mathrm{TiO}_{2}$ in supercritical water. J. Supercrit. Fluids 2018, 141, 173-181. [CrossRef]

54. Gong, Y.; Guo, Y.; Wang, S.; Song, W. Supercritical water oxidation of Quinazoline: Effects of conversion parameters and reaction mechanism. Water Res. 2016, 100, 116-125. [CrossRef]

55. Chen, Z.; Tong, K.; Xu, F.; Xue, M.; Chen, H.; Chen, Q.; Wang, D.; Xu, Y. Development of supercritical water oxidation technology for application to hazardous waste treatment: An extreme case study. J. Environ. Chem. Eng. 2021, 9, 105296. [CrossRef]

56. Kennedy, G.C. Pressure-volume-temperature relations in water at elevated temperatures and pressures. Am. J. Sci. 1950, 248, 540-564. [CrossRef]

57. Xu, X.; Antal, M.J. Gasification of Sewage Sludge and Other Biomass for Hydrogen Production in Supercritical Water. Environ. Prog. 1998, 17, 215-220. [CrossRef]

58. Kipçak, E.; Akgün, M. Oxidative gasification of olive mill wastewater as a biomass source in supercritical water: Effects on gasification yield and biofuel composition. J. Supercrit. Fluids 2012, 69, 57-63. [CrossRef]

59. Civan, F.; Özaltun, D.H.; Kipçak, E.; Akgün, M. The treatment of landfill leachate over $\mathrm{Ni} / \mathrm{Al}_{2} \mathrm{O}_{3}$ by supercritical water oxidation. J. Supercrit. Fluids 2015, 100, 7-14. [CrossRef] 
60. Miller, A.; Espanani, R.; Junker, A.; Hendry, D.; Wilkinson, N.; Bollinger, D.; Abelleira-Pereira, J.M.; Deshusses, M.A.; Inniss, E.; Jacoby, W. Supercritical water oxidation of a model fecal sludge without the use of a co-fuel. Chemosphere 2015, 141, 189-196. [CrossRef] [PubMed]

61. Peterson, A.A.; Dreher, M.; Wambach, J.; Nachtegaal, M.; Dahl, S.; Nørskov, J.K.; Vogel, F. Evidence of Scrambling over Ruthenium-based Catalysts in Supercritical-water Gasification. ChemCatChem 2012, 4, 1185-1189. [CrossRef]

62. Zhai, Y.; Wang, C.; Chen, H.; Li, C.; Zeng, G.; Pang, D.; Lu, P. Digested sewage sludge gasification in supercritical water. Waste Manag. Res. 2013, 31, 393-400. [CrossRef]

63. Güngören Madenoğlu, T.; Sağlam, M.; Yüksel, M.; Ballice, L. Hydrothermal gasification of biomass model compounds (cellulose and lignin alkali) and model mixtures. J. Supercrit. Fluids 2016, 115, 79-85. [CrossRef]

64. Jia, L.; Yu, J.; Chen, Y.; Ning, P.; Guan, Q.; Gu, J.; Miao, R.; Chen, Q. Noble-metal-free bimetallic alloy nanoparticle-catalytic gasification of phenol in supercritical water. J. Supercrit. Fluids 2017, 126, 79-88. [CrossRef]

65. Yildirir, E.; Onwudili, J.A.; Williams, P.T. Catalytic Supercritical Water Gasification of Refuse Derived Fuel for High Energy Content Fuel Gas. Waste Biomass Valorization 2017, 8, 359-367. [CrossRef]

66. Nanda, S.; Reddy, S.N.; Hunter, H.N.; Vo, D.V.N.; Kozinski, J.A.; Gökalp, I. Catalytic subcritical and supercritical water gasification as a resource recovery approach from waste tires for hydrogen-rich syngas production. J. Supercrit. Fluids $2019,154,104627$. [CrossRef]

67. Taufiq-Yap, Y.H.; Sivasangar, S.; Surahim, M. Catalytic Supercritical Water Gasification of Empty Palm Fruit Bunches Using ZnO-Doped Ni-CaO Catalyst for Hydrogen Production. Bioenergy Res. 2019, 12, 1066-1076. [CrossRef]

68. Okolie, J.A.; Mukherjee, A.; Nanda, S.; Dalai, A.K.; Kozinski, J.A. Catalytic Supercritical Water Gasification of Soybean Straw: Effects of Catalyst Supports and Promoters. Ind. Eng. Chem. Res. 2021, 60, 5770-5782. [CrossRef]

69. Jin, H.; Zhao, X.; Su, X.; Zhu, C.; Cao, C.; Guo, L. Supercritical water synthesis of bimetallic catalyst and its application in hydrogen production by furfural gasification in supercritical water. Int. J. Hydrogen Energy 2017, 42, 4943-4950. [CrossRef]

70. Liu, J.; Wang, D.; Yu, C.; Jiang, J.; Guo, M.; Hantoko, D.; Yan, M. A two-step process for energy-efficient conversion of food waste via supercritical water gasification: Process design, products analysis, and electricity evaluation. Sci. Total Environ. 2021, 752, 142331. [CrossRef]

71. Louw, J.; Schwarz, C.E.; Burger, A.J. Catalytic supercritical water gasification of primary paper sludge using a homogeneous and heterogeneous catalyst: Experimental vs thermodynamic equilibrium results. Bioresour. Technol. 2016, 201, 111-120. [CrossRef]

72. Cao, C.; Zhang, Y.; Cao, W.; Jin, H.; Guo, L.; Huo, Z. Transition Metal Oxides as Catalysts for Hydrogen Production from Supercritical Water Gasification of Glucose. Catal. Letters 2017, 147, 828-836. [CrossRef]

73. Chen, J.; Fan, Y.; Jiaqiang, E.; Cao, W.; Zhang, F.; Gong, J.; Liu, G.; Xu, W. Effects analysis on the gasification kinetic characteristics of food waste in supercritical water. Fuel 2019, 241, 94-104. [CrossRef]

74. Wang, C.; Zhu, C.; Cao, W.; Wei, W.; Jin, H. Catalytic mechanism study on the gasification of depolymerizing slag in supercritical water for hydrogen production. Int. J. Hydrogen Energy 2021, 46, 2917-2926. [CrossRef]

75. Castello, D.; Rolli, B.; Kruse, A.; Fiori, L. Supercritical Water Gasification of Biomass in a Ceramic Reactor: Long-Time Batch Experiments. Energies 2017, 10, 1734. [CrossRef]

76. Byrd, A.J.; Pant, K.K.; Gupta, R.B. Hydrogen production from ethanol by reforming in supercritical water using $\mathrm{Ru} / \mathrm{Al}_{2} \mathrm{O}_{3}$ catalyst. Energy Fuels 2007, 21, 3541-3547. [CrossRef]

77. Lee, I.G.; Ihm, S.K. Catalytic gasification of glucose over Ni/activated charcoal in supercritical water. Ind. Eng. Chem. Res. 2009, 48, 1435-1442. [CrossRef]

78. Qinming, Z.; Shuzhong, W.; Liang, W.; Donghai, X.; Lingling, L. Hydrogen production from sludge in supercritical water and its energy evaluation. In Proceedings of the 5th International Energy Conversion Engineering Conference and Exhibit (IECEC), San Diego, CA, USA, 25-27 June 2007; Volume 2, pp. 917-925. [CrossRef]

79. Stucki, S.; Vogel, F.; Ludwig, C.; Haiduc, A.G.; Brandenberger, M. Catalytic gasification of algae in supercritical water for biofuel production and carbon capture. Energy Environ. Sci. 2009, 2, 535-541. [CrossRef]

80. De Vlieger, D.J.M.; Chakinala, A.G.; Lefferts, L.; Kersten, S.R.A.; Seshan, K.; Brilman, D.W.F. Hydrogen from ethylene glycol by supercritical water reforming using noble and base metal catalysts. Appl. Catal. B Environ. 2012, 111-112, 536-544. [CrossRef]

81. Castello, D.; Kruse, A.; Fiori, L. Low temperature supercritical water gasification of biomass constituents: Glucose/phenol mixtures. Biomass Bioenergy 2015, 73, 84-94. [CrossRef]

82. Pinkard, B.R.; Gorman, D.J.; Tiwari, K.; Rasmussen, E.G.; Kramlich, J.C.; Reinhall, P.G.; Novosselov, I.V. Supercritical water gasification: Practical design strategies and operational challenges for lab-scale, continuous flow reactors. Heliyon 2019, 5 , e01269. [CrossRef]

83. Rorrer, G.L.; Goodwin, A.; Paul, B. Conversion of glucose to hydrogen gas by supercritical water within a microchannel reactor. Proceedingsof th The 2006 Annual Meeting, San Francisco, CA, 12-17 November 2006; pp. 4106-4114.

84. Goodwin, A.K.; Rorrer, G.L. Conversion of xylose and xylose-phenol mixtures to hydrogen-rich gas by supercritical water in an isothermal microtube flow reactor. Energy Fuels 2009, 23, 3818-3825. [CrossRef]

85. Rapagnà, S.; Jand, N.; Foscolo, P.U. Catalytic gasification of biomass to produce hydrogen rich gas. Int. J. Hydrogen Energy 1998, 23, 551-557. [CrossRef]

86. Cao, W.; Cao, C.; Guo, L.; Jin, H.; Dargusch, M.; Bernhardt, D.; Yao, X. Hydrogen production from supercritical water gasification of chicken manure. Int. J. Hydrogen Energy 2016, 41, 22722-22731. [CrossRef] 
87. Hantoko, D.; Su, H.; Yan, M.; Kanchanatip, E.; Susanto, H.; Wang, G.; Zhang, S.; Xu, Z. Thermodynamic study on the integrated supercritical water gasification with reforming process for hydrogen production: Effects of operating parameters. Int. J. Hydrogen Energy 2018, 43, 17620-17632. [CrossRef]

88. Fiori, L.; Castello, D. Thermodynamic Analysis of the Supercritical Water Gasification of Biomass. In Near-critical and Supercritical Water and Their Applications for Biorefineries; Fang, Z., Xu, C., Eds.; Springer: Berlin/Heidelberg, Germany, 2014; pp. 99-129.

89. Gutiérrez Ortiz, F.J.; Ollero, P.; Serrera, A.; Sanz, A. Thermodynamic study of the supercritical water reforming of glycerol. Int. J. Hydrogen Energy 2011, 36, 8994-9013. [CrossRef]

90. Gidner, A.; Stenmark, L. Supercritical water oxidation of sewage sludge-state of art. In Proceedings of the IBC's Conference on Sewage Sludge and Disposal Options, Birmingham, UK, 26-27 March 2000; pp. 1-16.

91. Kritzer, P.; Dinjus, E. An assessment of supercritical water oxidation (SCWO). Chem. Eng. J. 2001, 83, 207-214. [CrossRef]

92. Modell, M. Processing Methods for the Oxidation of Organics in Supercritical. Water. Patent US4338199A, 6 July 1982.

93. García-Jarana, M.B.; Kings, I.; Sánchez-Oneto, J.; Portela, J.R.; Al-Duri, B. Supercritical water oxidation of nitrogen compounds with multi-injection of oxygen. J. Supercrit. Fluids 2013, 80, 23-29. [CrossRef]

94. Al-Duri, B.; Alsoqyani, F.; Kings, I. Supercritical water oxidation for the destruction of hazardous waste: Better than incineration. Philos. Trans. R. Soc. A Math. Phys. Eng. Sci. 2015, 373, 20150013. [CrossRef] [PubMed]

95. Chen, Z.; Wang, G.; Yin, F.; Chen, H.; Xu, Y. A new system design for supercritical water oxidation. Chem. Eng. J. 2015, 269, 343-351. [CrossRef]

96. Vadillo, V.; García-Jarana, M.B.; Sánchez-Oneto, J.; Portela, J.R.; Martínez De La Ossa, E.J. New feed system for water-insoluble organic and/or highly concentrated wastewaters in the supercritical water oxidation process. J. Supercrit. Fluids 2012, 72, 263-269. [CrossRef]

97. Aki, S.N.V.K.; Ding, Z.Y.; Abraham, M.A. Catalytic Supercritical Water Oxidation: Stability of $\mathrm{Cr}_{2} \mathrm{O}_{3}$ Catalyst. AIChE J. 1996, 42, 1995-2004. [CrossRef]

98. OSHIMA, Y.; INABA, K.; KODA, S. Catalytic Supercritical Water Oxidation of Coke Works Waste with Manganese Oxide. J. Japan Pet. Inst. 2001, 44, 343-350. [CrossRef]

99. Smith, R.L.; Adschiri, T.; Arai, K. Energy integration of methane's partial-oxidation in supercritical water and exergy analysis. Appl. Energy 2002, 71, 205-214. [CrossRef]

100. Lavric, E.D.; Weyten, H.; De Ruyck, J.; Pleşu, V.; Lavric, V. Delocalized organic pollutant destruction through a self-sustaining supercritical water oxidation process. Energy Convers. Manag. 2005, 46, 1345-1364. [CrossRef]

101. Vadillo, V.; Sánchez-Oneto, J.; Portela, J.R.; Martínez de la Ossa, E.J. Chapter 9—Supercritical Water Oxidation for Wastewater Destruction with Energy Recovery. In Supercritical Fluid Technology for Energy and Environmental Applications; Elsevier: Amsterdam, The Netherlands, 2014; pp. 181-190. [CrossRef]

102. Abelleira, J.; Sánchez-Oneto, J.; Portela, J.R.; Martínez De La Ossa, E.J. Kinetics of supercritical water oxidation of isopropanol as an auxiliary fuel and co-fuel. Fuel 2013, 111, 574-583. [CrossRef]

103. Vadillo, V.; Sánchez-Oneto, J.; Portela, J.R.; Martínez De La Ossa, E.J. Problems in supercritical water oxidation process and proposed solutions. Ind. Eng. Chem. Res. 2013, 52, 7617-7629. [CrossRef]

104. Xu, D.; Wang, S.; Tang, X.; Gong, Y.; Guo, Y.; Zhang, J.; Wang, Y.; Ma, H.; Zhou, L. Influence of oxidation coefficient on product properties in sewage sludge treatment by supercritical water. Int. J. Hydrogen Energy 2013, 38, 1850-1858. [CrossRef]

105. Akgün, M.; Söğüt, O.Ö. Supercritical Water Oxidation (SCWO) for Wastewater Treatment. In Near-critical and Supercritical Water and Their Applications for Biorefineries; Fang, Z., Xu, C., Eds.; Springer: Berlin/Heidelberg, Germany, 2014; pp. $261-298$.

106. Wei, N.; Xu, D.; Hao, B.; Guo, S.; Guo, Y.; Wang, S. Chemical reactions of organic compounds in supercritical water gasification and oxidation. Water Res. 2021, 190, 116634. [CrossRef]

107. Krajnc, M.; Levec, J. Oxidation of Phenol over a Transition-Metal Oxide Catalyst in Supercritical Water. Ind. Eng. Chem. Res. 1997, 36, 3439-3445. [CrossRef]

108. Zhang, X.; Savage, P.E. Fast catalytic oxidation of phenol in supercritical water. Catal. Today 1998, 40, 333-342. [CrossRef]

109. Zhang, J.; Gu, J.; Han, Y.; Li, W.; Gan, Z.; Gu, J. Supercritical water oxidation vs supercritical water gasification: Which process is better for explosive wastewater treatment? Ind. Eng. Chem. Res. 2015, 54, 1251-1260. [CrossRef]

110. Thakur, S.; Kumar, A.; Reddy, S.N. Hydrothermal treatment of pharmaceutical wastewater. Indian Chem. Eng. 2019, 61, 415-423. [CrossRef]

111. Ding, N.; Azargohar, R.; Dalai, A.K.; Kozinski, J.A. Catalytic gasification of cellulose and pinewood to $\mathrm{H}_{2}$ in supercritical water. Fuel 2014, 118, 416-425. [CrossRef]

112. Norouzi, O.; Safari, F.; Jafarian, S.; Tavasoli, A.; Karimi, A. Hydrothermal gasification performance of Enteromorpha intestinalis as an algal biomass for hydrogen-rich gas production using $\mathrm{Ru}$ promoted $\mathrm{Fe}-\mathrm{Ni} / \Gamma-\mathrm{Al}_{2} \mathrm{O}_{3}$ nanocatalysts. Energy Convers. Manag. 2017, 141, 63-71. [CrossRef]

113. Guan, Q.; Wei, C.; Savage, P.E. Hydrothermal Gasification of Nannochloropsis sp. with Ru/C. Energy Fuels 2012, $26,4575-4582$. [CrossRef]

114. Peng, G.; Gramm, F.; Ludwig, C.; Vogel, F. Effect of carbon surface functional groups on the synthesis of Ru/C catalysts for supercritical water gasification. Catal. Sci. Technol. 2015, 5, 3658-3666. [CrossRef]

115. Yanik, J.; Ebale, S.; Kruse, A.; Saglam, M.; Yüksel, M. Biomass gasification in supercritical water: II. Effect of catalyst. Int. J. Hydrogen Energy 2008, 33, 4520-4526. [CrossRef] 
116. Rana, R.; Nanda, S.; Reddy, S.N.; Dalai, A.K.; Kozinski, J.A.; Gökalp, I. Catalytic gasification of light and heavy gas oils in supercritical water. J. Energy Inst. 2020, 93, 2025-2032. [CrossRef]

117. Yamaguchi, A.; Hiyoshi, N.; Sato, O.; Bando, K.K.; Osada, M.; Shirai, M. Hydrogen production from woody biomass over supported metal catalysts in supercritical water. Catal. Today 2009, 146, 192-195. [CrossRef]

118. Guo, Y.; Wang, S.; Yeh, T.; Savage, P.E. Catalytic gasification of indole in supercritical water. Appl. Catal. B Environ. 2015, 166-167, 202-210. [CrossRef]

119. Chen, Y.; Yi, L.; Li, S.; Yin, J.; Jin, H. Catalytic gasification of sewage sludge in near and supercritical water with different catalysts. Chem. Eng. J. 2020, 388, 124292. [CrossRef]

120. Nanda, S.; Reddy, S.N.; Dalai, A.K.; Kozinski, J.A. Subcritical and supercritical water gasification of lignocellulosic biomass impregnated with nickel nanocatalyst for hydrogen production. Int. J. Hydrogen Energy 2016, 41, 4907-4921. [CrossRef]

121. Nanda, S.; Reddy, S.N.; Vo, D.-V.N.; Sahoo, B.N.; Kozinski, J.A. Catalytic gasification of wheat straw in hot compressed (subcritical and supercritical) water for hydrogen production. Energy Sci. Eng. 2018, 6, 448-459. [CrossRef]

122. Su, H.; Kanchanatip, E.; Wang, D.; Zhang, H.; Antoni; Mubeen, I.; Huang, Z.; Yan, M. Catalytic gasification of food waste in supercritical water over La promoted $\mathrm{Ni} / \mathrm{Al}_{2} \mathrm{O}_{3}$ catalysts for enhancing $\mathrm{H}_{2}$ production. Int. J. Hydrogen Energy 2020, 45, 553-564. [CrossRef]

123. Nanda, S.; Rana, R.; Hunter, H.N.; Fang, Z.; Dalai, A.K.; Kozinski, J.A. Hydrothermal catalytic processing of waste cooking oil for hydrogen-rich syngas production. Chem. Eng. Sci. 2019, 195, 935-945. [CrossRef]

124. Kou, J.; Xu, J.; Jin, H.; Guo, L.; Zhang, D.; Cao, W. Evaluation of modified Ni/ZrO 2 catalysts for hydrogen production by supercritical water gasification of oil-containing wastewater. Int. J. Hydrogen Energy 2018, 43, 13896-13903. [CrossRef]

125. Rana, R.; Nanda, S.; Kozinski, J.A.; Dalai, A.K. Investigating the applicability of Athabasca bitumen as a feedstock for hydrogen production through catalytic supercritical water gasification. J. Environ. Chem. Eng. 2018, 6, 182-189. [CrossRef]

126. Tushar, M.S.H.K.; DiMaria, P.C.; Al-Salem, S.M.; Dutta, A.; Xu, C.C. Biohydrogen Production by Catalytic Supercritical Water Gasification: A Comparative Study. ACS Omega 2020, 5, 15390-15401. [CrossRef] [PubMed]

127. Boucard, H.; Watanabe, M.; Takami, S.; Weiss-Hortala, E.; Barna, R.; Adschiri, T. Beneficial use of $\mathrm{CeO}_{2}$ nanocatalyst for black liquor conversion under sub and supercritical conditions. J. Supercrit. Fluids 2015, 105, 66-76. [CrossRef]

128. Cao, C.; Xie, Y.; Mao, L.; Wei, W.; Shi, J.; Jin, H. Hydrogen production from supercritical water gasification of soda black liquor with various metal oxides. Renew. Energy 2020, 157, 24-32. [CrossRef]

129. Borges, A.C.P.; Onwudili, J.A.; Andrade, H.; Alves, C.; Ingram, A.; de Melo, S.V.; Torres, E. Catalytic properties and recycling of $\mathrm{NiFe} 2 \mathrm{O} 4$ catalyst for hydrogen production by supercritical water gasification of eucalyptus wood chips. Energies 2020, $13,4553$. [CrossRef]

130. Dong, X.; Gan, Z.; Lu, X.; Jin, W.; Yu, Y.; Zhang, M. Study on catalytic and non-catalytic supercritical water oxidation of p-nitrophenol wastewater. Chem. Eng. J. 2015, 277, 30-39. [CrossRef]

131. Ates, A.; Azimi, G.; Choi, K.-H.; Green, W.H.; Timko, M.T. The role of catalyst in supercritical water desulfurization. Appl. Catal. B Environ. 2014, 147, 144-155. [CrossRef]

132. Peng, G.; Vogel, F.; Refardt, D.; Ludwig, C. Catalytic Supercritical Water Gasification: Continuous Methanization of Chlorella vulgaris. Ind. Eng. Chem. Res. 2017, 56, 6256-6265. [CrossRef]

133. Tapah, B.F.; Santos, R.C.D.; Leeke, G.A. Processing of glycerol under sub and supercritical water conditions q. Renew. Energy 2014, 62, 353-361. [CrossRef]

134. Dong, X.; Zhang, Y.; Xu, Y.; Zhang, M. Catalytic mechanism study on manganese oxide in the catalytic supercritical water oxidation of nitrobenzene. RSC Adv. 2015, 5, 47488-47497. [CrossRef]

135. Li, S.; Zhu, B.; Wang, W.; Zhang, H.; Li, Q. Efficient and stable supercritical-water-synthesized Ni-based catalysts for supercritical water gasification. J. Supercrit. Fluids 2020, 160, 104790. [CrossRef]

136. Shirai, M.; Hiyoshi, N.; Murakami, Y.; Osada, M.; Sato, O.; Yamaguchi, A. Supercritical water gasification of organosolv lignin over a graphite-supported ruthenium metal catalyst. Chem. Lett. 2012, 41, 1453-1455. [CrossRef]

137. Lu, Y.; Zhu, Y.; Li, S.; Zhang, X.; Guo, L. Behavior of nickel catalysts in supercritical water gasification of glucose: Influence of support. Biomass Bioenergy 2014, 67, 125-136. [CrossRef] 